\title{
SÉRIES HYPERGÉOMÉTRIQUES BASIQUES, $q$-ANALOGUES DES VALEURS DE LA FONCTION ZÊTA ET SÉRIES D’EISENSTEIN
}

\author{
C. KRATTENTHALER ${ }^{1}$, T. RIVOAL ${ }^{2 *}$ ET W. ZUDILIN ${ }^{3}$ \\ ${ }^{1}$ Institut Camille Jordan, Université Claude Bernard Lyon-I, 21, avenue Claude \\ Bernard, F-69622 Villeurbanne Cedex, France (kratt@igd.univ-lyon1.fr) \\ ${ }^{2}$ Laboratoire de Mathématiques Nicolas Oresme, CNRS UMR 6139, Université de \\ Caen, BP 5186, 14032 Caen Cedex, France (rivoal@math.unicaen.fr) \\ ${ }^{3}$ Université d'État Lomonosov, Moscou, Faculté de Mécanique et Mathématiques, \\ Vorobiovy Gory, GSP-2, Moscou 119992, Russie (wadim@ips.ras.ru)
}

(Received 4 November 2003; accepted 3 March 2004)

Résumé Nous étudions la nature arithmétique de $q$-analogues des valeurs $\zeta(s)$ de la fonction zêta de Riemann, notamment des valeurs des fonctions $\zeta_{q}(s)=\sum_{k=1}^{\infty} q^{k} \sum_{d \mid k} d^{s-1}, s=1,2, \ldots$, où $q$ est un nombre complexe, $|q|<1$ (ces fonctions sont intimement liées au monde automorphe). Le théorème principal de cet article montre que, si $1 / q$ est un nombre entier différent de \pm 1 et si $M$ est un nombre impair suffisamment grand, alors la dimension de l'espace vectoriel engendré sur $\mathbb{Q}$ par $1, \zeta_{q}(3), \zeta_{q}(5), \ldots, \zeta_{q}(M)$ est au moins $c_{1} \sqrt{M}$, avec $c_{1}=0,3358$. Ce résultat peut être considéré comme un $q$-analogue du résultat de Rivoal et de Ball et Rivoal, qui affirme que la dimension de l'espace vectoriel engendré sur $\mathbb{Q}$ par $1, \zeta(3), \zeta(5), \ldots, \zeta(M)$ est au moins $c_{2} \log M$, avec $c_{2}=0,5906$. Pour les mêmes valeurs de $q$, une minoration similaire pour les valeurs $\zeta_{q}(s)$ aux entiers $s$ pairs nous permet de redémontrer un cas particulier d'un résultat de Bertrand qui affirme la transcendance sur $\mathbb{Q}$ de l'une des deux séries d'Eisenstein $E_{4}(q)$ et $E_{6}(q)$ pour tout nombre complexe $q$ tel que $0<|q|<1$.

Abstract We study the arithmetic properties of $q$-analogues of values $\zeta(s)$ of the Riemann zeta function, in particular of the values of the functions $\zeta_{q}(s)=\sum_{k=1}^{\infty} q^{k} \sum_{d \mid k} d^{s-1}, s=1,2, \ldots$, where $q$ is a complex number with $|q|<1$ (these functions are also connected with the automorphic world). The main theorem of this article is that, if $1 / q$ is an integer different from \pm 1 , and if $M$ is a sufficiently large odd integer, then the dimension of the vector space over $\mathbb{Q}$ which is spanned by $1, \zeta_{q}(3), \zeta_{q}(5), \ldots, \zeta_{q}(M)$ is at least $c_{1} \sqrt{M}$, where $c_{1}=0.3358$. This result can be regarded as a $q$-analogue of the result of Rivoal and of Ball and Rivoal that the dimension of the vector space over $\mathbb{Q}$ which is spanned by $1, \zeta(3), \zeta(5), \ldots, \zeta(M)$ is at least $c_{2} \log M$, with $c_{2}=0.5906$. For the same values of $q$, a similar lower bound for the values $\zeta_{q}(s)$ at even integers $s$ provides a new proof of a special case of a result of Bertrand saying that one of the two Eisenstein series $E_{4}(q)$ and $E_{6}(q)$ is transcendental over $\mathbb{Q}$ for any complex number $q$ such that $0<|q|<1$.

Mots clés : q-analogues de la fonction zêta de Riemann; formes modulaires; séries d'Eisenstein; séries hypergéométriques basiques

Keywords: q-analogues of the Riemann zeta function; modular forms; Eisenstein series; basic hypergeometric series

AMS $2000 M S C$ : Primaire 11J72

Secondaire 11M36; 33D15

* Adresse actuelle : Institut Fourier, CNRS UMR 5582, Université Grenoble 1, 100 rue des Maths, BP 74, F-38402 Saint-Martin d'Hères cedex, France. 


\section{Introduction et énoncés des résultats}

L'étude arithmétique des valeurs aux entiers $\geqslant 2$ de la fonction zêta de Riemann est un sujet classique de la théorie analytique des nombres. Pour les entiers $2 m$ pairs, on connait la formule d'Euler

$$
\zeta(2 m)=(-1)^{m-1} \frac{2^{2 m-1} B_{2 m}}{(2 m) !} \pi^{2 m},
$$

où le rationnel $B_{m}$ est le mième nombre de Bernoulli, ce qui, joint au fait que $\pi$ est transcendant, établit que $\zeta(2 m)$ l'est aussi pour tout entier $m \geqslant 1$. Concernant les valeurs $\zeta(2 m+1), m \geqslant 1$, notre connaissance est beaucoup plus faible et pendant longtemps, le seul résultat connu a été le célèbre théorème d'Apéry $[\mathbf{1}] \ll \zeta(3)$ est irrationnel $»$. Récemment, plusieurs résultats nouveaux sur les $\zeta(2 m+1)$ ont été démontrés (voir $[\mathbf{2 1}],[\mathbf{2}],[\mathbf{2 3}],[\mathbf{2 2}],[\mathbf{3 4}]$ et $[\mathbf{9}]$ pour un survol de ce sujet), notamment, le théorème suivant dans $[\mathbf{2}, \mathbf{2 1}]$. (Seule est montrée la minoration aux entiers impairs dans les deux références citées ; celle aux entiers pairs est immédiate avec la méthode utilisée.)

Théorème 1.1. Pour tout entier A pair suffisamment grand, on a

$$
\operatorname{dim}_{\mathbb{Q}}(\mathbb{Q}+\mathbb{Q} \zeta(3)+\mathbb{Q} \zeta(5)+\cdots+\mathbb{Q} \zeta(A-1)) \geqslant \frac{1+o(1)}{1+\log 2} \log A
$$

et

$$
\operatorname{dim}_{\mathbb{Q}}(\mathbb{Q}+\mathbb{Q} \zeta(2)+\mathbb{Q} \zeta(4)+\cdots+\mathbb{Q} \zeta(A)) \geqslant \frac{1+o(1)}{1+\log 2} \log A
$$

L'objet de cet article est d'étudier les propriétés arithmétiques de $q$-analogues des valeurs de fonction zêta de Riemann. En particulier, nous considérons les séries $\zeta_{q}(s)$, où $s \geqslant 1$ et $q$ est un nombre complexe tels que $|q|<1$, définies par

$$
\zeta_{q}(s)=\sum_{k=1}^{\infty} \sigma_{s-1}(k) q^{k}=\sum_{m=1}^{\infty} m^{s-1} \frac{q^{m}}{1-q^{m}}
$$

avec $\sigma_{s-1}(k)=\sum_{d \mid k} d^{s-1}$. Ces $q$-analogues normalisés de $\zeta(s)$ ont été considérés dans $[\mathbf{1 4}, \mathbf{3 1}]$, par exemple (voir aussi le paragraphe 4.1 pour la vérification que $\zeta_{q}(s)$ est effectivement un $q$-analogue de $\zeta(s))$. Plus précisément, notre but est de montrer les Théorèmes $1.2,1.3$ et 1.4 suivants.

Théorème 1.2. Fixons $q \neq \pm 1$ tel que $1 / q \in \mathbb{Z}$. Pour tout entier $A$ pair, on a

$$
\operatorname{dim}_{\mathbb{Q}}\left(\mathbb{Q}+\mathbb{Q} \zeta_{q}(3)+\mathbb{Q} \zeta_{q}(5)+\cdots+\mathbb{Q} \zeta_{q}(A-1)\right) \geqslant \frac{\pi+o(1)}{2 \sqrt{\pi^{2}+12}} \sqrt{A}
$$

et

$$
\operatorname{dim}_{\mathbb{Q}}\left(\mathbb{Q}+\mathbb{Q} \zeta_{q}(2)+\mathbb{Q} \zeta_{q}(4)+\cdots+\mathbb{Q} \zeta_{q}(A)\right) \geqslant \frac{\pi+o(1)}{2 \sqrt{\pi^{2}+12}} \sqrt{A} .
$$


La minoration de la dimension des valeurs de $\zeta_{q}(s)$ aux entiers pairs possède une intéressante traduction en terme de formes modulaires. Donnons d'abord quelques définitions (voir $[25]$ pour plus de détails). Posons $q=\mathrm{e}^{2 \mathrm{i} \pi \tau}$, où $\tau \in \mathcal{H}=\{\tau \in \mathbb{C}: \operatorname{Im}(\tau)>0\}$. Toute matrice $\gamma=\left(\begin{array}{ll}a & b \\ c & d\end{array}\right) \in S L_{2}(\mathbb{Z})$ agit sur $\mathcal{H}$ par $\gamma \cdot \tau=(a \tau+b) /(c \tau+d)$. Les séries d'Eisenstein $G_{2 s}(\tau)$ et $E_{2 s}(q)$ sont définies pour tout entier $s \geqslant 1$ par

$$
G_{2 s}(\tau)=2 \zeta(2 s) E_{2 s}(q)=\sum_{\substack{(m, n) \in \mathbb{Z}^{2} \\(m, n) \neq(0,0)}} \frac{1}{(m \tau+n)^{2 s}}
$$

et on a le développement en série de Fourier :

$$
E_{2 s}(q)=1-\frac{4 s}{B_{2 s}} \sum_{k=1}^{\infty} \sigma_{2 s-1}(k) q^{k} .
$$

On a donc $E_{2 s}(q)=1-\left(4 s / B_{2 s}\right) \zeta_{q}(2 s)$. Pour tout $s \geqslant 2$, les séries d'Eisenstein sont modulaires sur le groupe $S L_{2}(\mathbb{Z})$, de poids $2 s$, c'est-à-dire que pour tout $\gamma=\left(\begin{array}{ll}a & b \\ c & d\end{array}\right) \in S L_{2}(\mathbb{Z})$, on a $G_{2 s}(\gamma \cdot \tau)=(c \tau+d)^{2 s} G_{2 s}(\tau)$. La fonction $G_{2}(\tau)$ n'est pas modulaire. On a alors le théorème suivant.

Théorème 1.3. Pour tout $q \neq \pm 1$ tel que $1 / q \in \mathbb{Z}$, au moins un des deux nombres $E_{4}(q)$ et $E_{6}(q)$ est transcendant $\operatorname{sur} \mathbb{Q}$.

Ce résultat n'est pas nouveau puisque Bertrand [5] a montré que «pour tout $q \in \mathbb{C}$ tel que $0<|q|<1$, au moins un des nombres $E_{4}(q)$ et $E_{6}(q)$ est transcendant sur $\mathbb{Q} »$, comme conséquence d'un résultat de Schneider sur les fonctions elliptiques [24, Théorème 18, p. 64]. Le théorème stéphanois $[\mathbf{3}]$ précise le théorème de Bertrand : «lorsque $q$ est algébrique, l'invariant modulaire $J(q)=1728 E_{4}^{3}(q) /\left(E_{4}^{3}(q)-E_{6}^{2}(q)\right)$ est transcendant sur $\mathbb{Q}$ ». Le résultat définitif dans cette direction est le théorème de Nesterenko [18]: «pour tout $q \in \mathbb{C}$ tel que $0<|q|<1$, au moins trois des nombres $q, E_{2}(q), E_{4}(q), E_{6}(q)$ sont algébriquement indépendants sur $\mathbb{Q}$ ». Cependant, la démonstration du Théorème 1.3 est basée sur une fonction auxiliaire totalement explicite (notre $S_{n}^{[\epsilon]}(q)$ au paragraphe 3.1), et pas sur celles, beaucoup moins explicites, que l'on peut construire avec les outils diophantiens usuels tels le lemme de Siegel ou les déterminants d'interpolation de Laurent (voir [3], [18] et [19] pour l'utilisation de ces outils dans le contexte modulaire).

$A$ contrario, la minoration de la dimension des valeurs de $\zeta_{q}(s)$ aux entiers impairs est nouvelle et ne possède, à notre connaissance, aucune interprétation en terme des formes modulaires ci-dessus. Cependant, ces valeurs $\zeta_{q}(s)$, pour $s$ impair, ont malgré tout un lien avec le monde automorphe, via les séries d'Eisenstein non-holomorphes

$$
E_{s}(\tau)=\frac{1}{2} \sum_{\substack{(m, n) \in \mathbb{Z}^{2} \\(m, n) \neq(0,0)}} \frac{y^{s}}{|m \tau+n|^{2 s}}
$$

où $y=\operatorname{Im} \tau$. Ces séries sont des formes de Maass non paraboliques et sont invariantes sous l'action de $S L_{2}(\mathbb{Z})$. Sans rentrer dans les détails, indiquons seulement que dans 
[15, p. 243], Lewis et Zagier déterminent les fonctions périodes $\psi_{s}^{+}(\tau)$ des $E_{s}(\tau)$ : pour $s=2 \ell+1$, les valeurs $\zeta_{q}(s)$ sont essentiellement égales à $\psi_{\ell+1 / 2}^{+}(\tau)+\tau^{-2 \ell-1} \psi_{\ell+1 / 2}^{+}(-1 / \tau)$.

Du point de vue diophantien, si l'irrationalité de $\zeta_{q}(1)$ est connue pour diverses valeurs de $q$ (voir $[\mathbf{7}]$ et les références données dans $[\mathbf{2 9}, \mathbf{3 3}]$ ), celle de $\zeta_{q}(2 \ell+1)($ pour $\ell \geqslant 1)$ ne l'est pour aucune valeur de $q$, même si la transcendance de ces valeurs comme fonctions de $q$ est connue (voir [32]). Remarquons que pour $1 / q \in \mathbb{Z}, q \neq \pm 1$, des mesures d'irrationalité de $\zeta_{q}(1)$ et $\zeta_{q}(2)$ sont aussi données par le dernier auteur dans $[\mathbf{3 0}, \mathbf{3 3}]$ et que Postelmans et Van Assche [20] ont récemment démontré l'indépendance linéaire de $1, \zeta_{q}(1)$ et $\zeta_{q}(2)$. Enfin, une minoration de dimension dans le contexte différent de la fonction $q$-exponentielle se trouve dans [8].

Compte-tenu de la formule d'Euler (1.1), la minoration de la dimension de l'espace des $\zeta$ pairs est équivalente à la transcendance* de $\pi$ : on peut donc considérer que les Théorèmes 1.2 et 1.3 sont des $q$-analogues respectifs du Théorème 1.1 et de la transcendance de $\pi$. Comme on le verra au paragraphe 4.1, lorsque l'on fait tendre $q$ vers 1 , notre construction «tend » vers celle utilisée pour montrer le Théorème 1.1. Puis, on présente au paragraphe 4.2 des $q$-analogues de certaines séries hypergéométriques apparues dans diverses démonstrations de l'irrationalité de $\zeta(3)$. Dans ce contexte, notre $q$-analogue de $\zeta(3)$, à savoir $\zeta_{q}(3)$, apparaît naturellement, mais, malheureusement, nous ne réussissons à démontrer son irrationalité pour aucune valeur de $q$.

En utilisant les estimations précises utilisées pour montrer le Théorème 1.2, on peut cependant démontrer sans effort le théorème ci-dessous, de même facture que le résultat du dernier auteur $[\mathbf{3 4}]:$ « au moins un des nombres $\zeta(5), \zeta(7), \zeta(9), \zeta(11)$ est irrationnel».

Théorème 1.4. Pour tout $q \neq \pm 1$ tel que $1 / q \in \mathbb{Z}$, au moins un des nombres $\zeta_{q}(3)$, $\zeta_{q}(5), \zeta_{q}(7), \zeta_{q}(9), \zeta_{q}(11)$ est irrationnel.

Il est probable que l'on puisse améliorer ce résultat (i.e. démontrer qu'au moins un des nombres $\zeta_{q}(3), \zeta_{q}(5), \zeta_{q}(7), \zeta_{q}(9)$ est irrationnel) en utilisant une série légèrement différente de notre série $S_{n}^{[\epsilon]}(q)$ ci-dessous (voir la Remarque (2) au paragraphe 3.1), à condition que l'on puisse démontrer une certaine «conjecture des dénominateurs » pour cette série (voir la remarque à la fin de l'article).

\section{Démonstration du Théorème 1.3}

Le Théorème 1.3 est une conséquence du résultat suivant, dont on trouvera la démonstration dans $[\mathbf{2 5}]$.

Proposition 2.1 (Structure de l'espace des formes modulaires). Soit $M(q)$ une forme modulaire sur $S L_{2}(\mathbb{Z})$, holomorphe sur le disque $\{q \in \mathbb{C}:|q|<1\}$, de poids $n \geqslant 1$. Alors $n$ est pair et il existe des nombres complexes $c_{a, b}$ tels que

$$
M(q)=\sum c_{a, b} E_{4}(q)^{a} E_{6}(q)^{b},
$$

où la sommation est étendue à tous les couples d'entiers positifs $(a, b)$ tels que $4 a+6 b=n$.

\footnotetext{
* Pour le voir, il suffit d'adapter l'argument utilisé au paragraphe 2.
} 
Lorsque $M(q)$ est une série d'Eisenstein $E_{2 n}(q)$, les $c_{a, b}$ peuvent être pris rationnels. Comme pour tout entier pair $j \geqslant 4$, les formes modulaires $\zeta_{q}(j)$ de poids $j$ vérifient les hypothèses de ce théorème, on en déduit que pour tout $A$ pair, le $\mathbb{Q}$-espace vectoriel $V(A)$ engendré par $1, \zeta_{q}(4), \zeta_{q}(6), \ldots, \zeta_{q}(A)$ est inclus dans le $\mathbb{Q}$-espace vectoriel $W(A)$ engendré par les puissances $E_{4}(q)^{a} E_{6}(q)^{b}$, avec $0 \leqslant a \leqslant A / 4$ et $0 \leqslant b \leqslant A / 6$. Fixons $q \neq \pm 1$ tel que $1 / q \in \mathbb{Z}$; si les nombres $E_{4}(q)$ et $E_{6}(q)$ étaient tous les deux algébriques sur $\mathbb{Q}$, la dimension de $W(A)$, et donc celle de $V(A)$, serait bornée indépendamment de $A$. Or ceci est impossible puisque, pour $A$ assez grand, cela contredit la minoration de la dimension de $V(A)$ fournit par le Théorème 1.2. D'où le Théorème 1.3.

Remarque. D'après le théorème de Nesterenko $[\mathbf{1 8}]$, les nombres $\zeta_{q}(2), \zeta_{q}(4)$ et $\zeta_{q}(6)$ sont algébriquement indépendants pour $q \neq \pm 1$ tel que $1 / q \in \mathbb{Z}$. Pour tout $A \geqslant 2$ pair, on a donc en fait

$$
\operatorname{dim}_{\mathbb{Q}}\left(\mathbb{Q}+\mathbb{Q} \zeta_{q}(2)+\mathbb{Q} \zeta_{q}(4)+\cdots+\mathbb{Q} \zeta_{q}(A)\right)=A / 2+1 .
$$

\section{Démonstrations des Théorèmes 1.2 et 1.4}

\subsection{La méthode}

La démonstration est basée sur la construction de combinaisons linéaires rationnelles en 1 et les $\zeta_{q}(j)$, avec $j$ pair ou impair, exclusivement. Pour cela, introduisons la série

$$
S_{n}(q)=(q ; q)_{n}^{A-2 r} \sum_{k=1}^{\infty} q^{k} \frac{\left(q^{k-r n} ; q\right)_{r n}\left(q^{k+n+1} ; q\right)_{r n}}{\left(q^{k} ; q\right)_{n+1}^{A}} q^{(k-1 / 2)(A-2 r) n / 2},
$$

où les $q$-factoriels montants sont définis par $(\alpha ; q)_{m}=(1-\alpha)(1-q \alpha) \cdots\left(1-q^{m-1} \alpha\right)$ si $m \geqslant 1$, et $(\alpha ; q)_{0}=1$, et où l'on suppose $A, n, r$ entiers tels que

$$
n \geqslant 0, A \text { pair et } 1 \leqslant r \leqslant A / 2 .
$$

Cette série, qui converge pour tout nombre complexe $q$ tel que $|q| \neq 1$, est une série hypergéométrique basique (voir le paragraphe 3.3 pour plus de détails). Fixons $\epsilon \in\{0,1\}$ et $A, n, r$ vérifiant (3.1), et $q$ un nombre complexe tel que $|q|<1$. La série $S_{n}(q)$ vérifie les propriétés suivantes.

(A) Il existe des fractions rationnelles $P_{s, n}^{[\epsilon]}(q)$ de $\mathbb{Q}(q)$, avec $s \in\{2, \ldots, A\}$, telles que

$$
S_{n}^{[\epsilon]}(q)=S_{n}(q)+(-1)^{\epsilon} q^{-n} S_{n}(1 / q)=P_{0, n}^{[\epsilon]}(q)+\sum_{\substack{s=2 \\ s \equiv \epsilon(\bmod 2)}}^{A} P_{s, n}^{[\epsilon]}(q) \zeta_{q}(s) .
$$

(B) On a

$$
\lim _{n \rightarrow+\infty} \frac{1}{n^{2}} \log \left|S_{n}^{[\epsilon]}(q)\right|=-\frac{1}{2} r(A-2 r) \log |1 / q| \neq 0 .
$$


(C) Pour tout $s \in\{0, \ldots, A\}$, on a

$$
\limsup _{n \rightarrow+\infty} \frac{1}{n^{2}} \log \left|P_{s, n}^{[\epsilon]}(q)\right| \leqslant \frac{1}{8}\left(A+4 r^{2}\right) \log |1 / q| .
$$

(D) Il existe $D_{n}(q) \in \mathbb{Q}(q)$ tel que pour $s \in\{0, \ldots, A\}$ on a $D_{n}(q) P_{s, n}^{[\epsilon]}(q) \in \mathbb{Z}[1 / q]$ et

$$
\lim _{n \rightarrow+\infty} \frac{1}{n^{2}} \log \left|D_{n}(q)\right|=\left(\frac{3}{\pi^{2}} A+\frac{A}{8}+\frac{r^{2}}{2}\right) \log |1 / q| .
$$

Remarques. (1) La démonstration du point (D) montrera que l'estimation arithmétique donnée dans ce point est optimale, i.e. que l'on ne peut pas remplacer ce $D_{n}(q)$ par un $\tilde{D}_{n}(q)$ ayant les mêmes propriétés que $D_{n}(q)$, mais tel que la limite de $\left(1 / n^{2}\right) \log \left|\tilde{D}_{n}(q)\right|$ soit plus petite que le membre de droite de (3.2). Ceci s'applique probablement aussi à l'estimation donnée au point $(\mathrm{C})$ (voir la remarque à la fin du paragraphe 3.5). Par conséquent, la minoration des dimensions donnée au Théorème 1.2 est très certainement la meilleure que l'on puisse obtenir par la méthode utilisée et notre série $S_{n}^{[\epsilon]}(q)$.

(2) En revanche, il est sans doute possible d'améliorer certains des points (A) à (D) en utilisant d'autres séries et en raffinant la méthode. Par exemple, on peut obtenir les mêmes résultats $(A)-(D)$ en utilisant la série

$$
\tilde{S}_{n}(q)=(q ; q)_{n}^{A-2 r} \sum_{k=1}^{\infty}\left(1-q^{2 k+n}\right) \frac{\left(q^{k-r n} ; q\right)_{r n}\left(q^{k+n+1} ; q\right)_{r n}}{\left(q^{k} ; q\right)_{n+1}^{A}} q^{k((A-2 r) n / 2+A / 2-1)}
$$

en lieu et place de $S_{n}^{[\epsilon]}(q)$ et en appliquant des arguments similaires. Or cette série a semble-t-il certains avantages arithmétiques sur la série $S_{n}^{[\epsilon]}(q)$ (voir la remarque à la fin de l'article).

Le Théorème 1.2 résulte des propriétés $(\mathrm{A})-(\mathrm{D})$ ci-dessus et du cas particulier suivant d'un critère d'indépendance linéaire, dû à Nesterenko [16].

Proposition 3.1 (Critère d'indépendance linéaire). Soient un entier $N \geqslant 2$ et $\vartheta_{1}, \ldots, \vartheta_{N}$ des réels. Supposons qu'il existe $N$ suites d'entiers $\left(p_{j, n}\right)_{n \geqslant 0}$ et des réels $\alpha$ et $\beta$ avec $\beta>0$ tels que

(i) pour tout $j \in\{1, \ldots, N\}$, on a $\limsup _{n \rightarrow+\infty} \frac{1}{n^{2}} \log \left|p_{j, n}\right| \leqslant \beta$;

(ii) $\lim _{n \rightarrow+\infty} \frac{1}{n^{2}} \log \left|p_{1, n} \vartheta_{1}+\cdots+p_{N, n} \vartheta_{N}\right|=\alpha$.

Alors la dimension $d u \mathbb{Q}$-espace vectoriel engendré par $\vartheta_{1}, \ldots, \vartheta_{N}$ est plus grande que

$$
1-\frac{\alpha}{\beta} \text {. }
$$

Démonstration du Théorème 1.2. Supposons $q \neq \pm 1$ tel que $1 / q \in \mathbb{Z}$ et posons

$$
\mathfrak{P}_{s, n}^{[\epsilon]}(q)=D_{n}(q) P_{s, n}^{[\epsilon]}(q) \in \mathbb{Z}[1 / q] \subset \mathbb{Z} \quad \text { et } \quad \mathfrak{S}_{n}^{[\epsilon]}(q)=D_{n}(q) S_{n}^{[\epsilon]}(q) .
$$


En vertu du point (A) ci-dessus, on a

$$
\mathfrak{S}_{n}^{[\epsilon]}(q)=\mathfrak{P}_{0, n}^{[\epsilon]}(q)+\sum_{\substack{s=2 \\ s \equiv \epsilon(\bmod 2)}}^{A} \mathfrak{P}_{s, n}^{[\epsilon]}(q) \zeta_{q}(s) .
$$

Grâce aux estimations résumées par les point (B) à (D) ci-dessus, la Proposition 3.1 appliquée à la combinaison linéaire $\mathfrak{S}_{n}^{[\epsilon]}(q)$ permet alors d'affirmer que pour tout $A \geqslant 2$ pair, les deux dimensions

$$
\operatorname{dim}_{\mathbb{Q}}\left(\mathbb{Q}+\mathbb{Q} \zeta_{q}(3)+\mathbb{Q} \zeta_{q}(5)+\cdots+\mathbb{Q} \zeta_{q}(A-1)\right)
$$

et

$$
\operatorname{dim}_{\mathbb{Q}}\left(\mathbb{Q}+\mathbb{Q} \zeta_{q}(2)+\mathbb{Q} \zeta_{q}(4)+\cdots+\mathbb{Q} \zeta_{q}(A)\right)
$$

sont minorées, pour tout $r \in\{1, \ldots, A / 2\}$, par

$$
\delta(A, r)=\frac{4 r A+A-4 r^{2}}{\left(\left(24 / \pi^{2}\right)+2\right) A+8 r^{2}} .
$$

On choisit alors $r$ de la forme $u \sqrt{A}$ où $u>0$; un calcul immédiat montre que

$$
\max _{u>0} \lim _{A \rightarrow+\infty} \frac{1}{\sqrt{A}} \delta(A, u \sqrt{A})=\max _{u>0}\left(\frac{4 u}{\left(24 / \pi^{2}\right)+2+8 u^{2}}\right)=\frac{\pi}{2 \sqrt{\pi^{2}+12}} \approx 0,335892 .
$$

Le Théorème 1.2 en découle.

Démonstration du Théorème 1.4. On répète la démonstration du Théorème 1.2. En choisissant $A=12$ et $r=2$ dans (3.4), on obtient $\delta(12,2)=1,080059 \cdots>1$. La dimension de l'espace vectoriel engendré sur $\mathbb{Q}$ par $1, \zeta_{q}(3), \zeta_{q}(5), \zeta_{q}(7), \zeta_{q}(9), \zeta_{q}(11)$ est donc au moins 2 .

Les points (A), (B), (C) et (D) sont démontrés respectivement aux Lemmes 3.4, 3.5, 3.6 et 3.7, dans les sous-paragraphes qui suivent. On fait la convention suivante : la valeur d'une fonction $F(z ; q)$ en $z=1$ sera notée indifféremment $F(1 ; q)$ ou $F(q)$, s'il n'y a pas d'ambiguité possible.

\subsection{Des fonctions intermédiaires}

On suppose dans tout ce paragraphe que $|q|<1$. Pour tout $s \geqslant 1$, la fonction $\mathcal{Z}_{s}(z ; q)$ est définie par la série

$$
\mathcal{Z}_{s}(z ; q)=\sum_{k=1}^{\infty} \frac{q^{k}}{\left(1-q^{k}\right)^{s}} z^{-k},
$$

qui converge pour tout $z$ tel que $|q|<|z|$ et en particulier en $z=1$. On aura besoin de considérer simultanément les fonctions $\mathcal{Z}_{s}(z ; q)$ et $\mathcal{Z}_{s}(1 / z ; 1 / q):$ si $s \geqslant 2$, alors cette 
dernière série est convergente dès que $|z|<|q|^{1-s}$ et il est donc possible de définir simultanément nos deux fonctions sur $|q|<|z|<|q|^{1-s}$ et en particulier toujours en $z=1$. Lorsque $s=1$, la série $\mathcal{Z}_{1}(1 / z ; 1 / q)$ converge sur $|z|<|q|^{1-s}=1$, et les deux fonctions $\mathcal{Z}_{1}(z ; q)$ et $\mathcal{Z}_{1}(1 / z ; 1 / q)$ sont donc définies simultanément sur $|q|<|z|<1$.

Sauf mention contraire, on supposera dans toute la suite de cet article que $|q|<|z|<1$. On fera tendre $z$ vers 1 pour définir une expression en $z=1$.

Avec cette condition, toutes les séries de ce paragraphe sont absolument convergentes et en développant $1 /\left(1-q^{k}\right)^{s}$ en série de puissances de $q^{k}$, on obtient pour tout $s \geqslant 1$ que

$$
\mathcal{Z}_{s}(z ; q)=\frac{1}{(s-1) !} \sum_{k=1}^{\infty} \sum_{\ell=1}^{\infty} \ell(\ell+1) \cdots(\ell+s-2) z^{-k} q^{k \ell}
$$

et

$$
\mathcal{Z}_{s}(1 / z ; 1 / q)=\frac{(-1)^{s}}{(s-1) !} \sum_{k=1}^{\infty} \sum_{\ell=0}^{\infty} \ell(\ell-1) \cdots(\ell-s+2) z^{k} q^{k \ell}
$$

en convenant que pour $s=1$ les produits vides $\ell(\ell+1) \cdots(\ell+s-2)$ et $\ell(\ell-1) \cdots(\ell-s+2)$ sont interprétés comme 1 . Les fonctions $\mathcal{Z}_{s}(z ; q)$ sont donc des combinaisons linéaires en les fonctions $\mathcal{L}_{j}(z ; q)$, définies par les séries (avec $j \geqslant 1$ ):

$$
\mathcal{L}_{j}(z ; q)=\sum_{k=1}^{\infty} \sum_{\ell=1}^{\infty} \ell^{j-1} z^{-k} q^{k \ell}=\sum_{k=1}^{\infty} \sigma_{j-1}(z ; k) q^{k} \quad \text { avec } \sigma_{j-1}(z ; k)=\sum_{d \mid k} d^{j-1} z^{-k / d}
$$

Plus précisément, si l'on utilise les nombres de Stirling de première espèce (sans signe) $c(N, j)$, définis par (voir $[\mathbf{2 6}, \S 1.3])$

$$
\ell(\ell+1) \cdots(\ell+N-1)=\sum_{j=1}^{N} c(N, j) \ell^{j},
$$

on a

$$
\mathcal{Z}_{s}(z ; q)=\frac{1}{(s-1) !} \sum_{j=2}^{s} c(s-1, j-1) \mathcal{L}_{j}(z ; q) .
$$

Évidemment, le coefficient de $\mathcal{L}_{j}(z ; q)$ est un nombre rationnel ne dépendant que de $s$ et $j$. De plus, en comparant les définitions $(1.2)$ et $(3.7)$, on remarque que $\mathcal{L}_{j}(1 ; q)=\zeta_{q}(j)$.

\section{Lemme 3.2.}

(i) Pour tout entier $s \geqslant 2$, on a

$$
\mathcal{Z}_{s}(q)-\mathcal{Z}_{s}(1 / q)=\frac{2}{(s-1) !} \sum_{\substack{j=3 \\ j \text { impair }}}^{s} c(s-1, j-1) \zeta_{q}(j)
$$


(ii) Pour tout entier $s \geqslant 2$, on a

$$
\mathcal{Z}_{s}(q)+\mathcal{Z}_{s}(1 / q)=\frac{2}{(s-1) !} \sum_{\substack{j=2 \\ j \text { pair }}}^{s} c(s-1, j-1) \zeta_{q}(j)
$$

(iii) On a $\mathcal{Z}_{1}(z ; q)=\mathcal{L}_{1}(z ; q)$ et $\mathcal{Z}_{1}(1 / z ; 1 / q)=-\mathcal{L}_{1}(1 / z ; q)-z /(1-z)$.

Preuve. (i), (ii) Supposons $s \geqslant 2$. Comme les séries $\mathcal{Z}_{s}(z ; q)$ et $\mathcal{Z}_{s}(1 / z ; 1 / q)$ sont convergentes en $z=1$, on a, en vertu de (3.5) et (3.6),

$$
\mathcal{Z}_{s}(q)+(-1)^{\epsilon} \mathcal{Z}_{s}(1 / q)=\frac{1}{(s-1) !} \sum_{k=1}^{\infty} \sum_{\ell=1}^{\infty}\left((\ell)_{s-1}+(-1)^{1+\epsilon}(-\ell)_{s-1}\right) q^{k \ell}
$$

où le symbole de Pochhammer $(\alpha)_{m}$ est défini par $(\alpha)_{m}=\alpha(\alpha+1) \cdots(\alpha+m-1)$, $m \geqslant 1$, et $(\alpha)_{0}=1$. Il est justifié de débuter la sommation sur $\ell$ à partir de 1 puisque pour $s \geqslant 2$, on a $(0)_{s-1}=0$. Si $\epsilon=0$, respectivement 1 , alors $(\ell)_{s-1}+(-1)^{1+\epsilon}(-\ell)_{s-1}$ est un polynôme impair, respectivement pair. Cela démontre (i) et (ii), sauf qu'il reste l'éventualité que $\zeta_{q}(1)$ apparaisse aussi dans la combinaison linéaire pour $\epsilon=1$ : comme $(0)_{s-1}=0$, c'est en fait impossible. Les formes explicites (3.10) et (3.11) résultent de (3.8).

(iii) En utilisant (3.5), on obtient

$$
\mathcal{Z}_{1}(z ; q)=\sum_{k=1}^{\infty} \sum_{\ell=1}^{\infty} z^{-k} q^{k \ell}=\mathcal{L}_{1}(z ; q)
$$

De même, en utilisant (3.6), on obtient

$$
\mathcal{Z}_{1}(1 / z ; 1 / q)=-\sum_{k=1}^{\infty} \sum_{\ell=0}^{\infty} z^{k} q^{k \ell}=-\sum_{k=1}^{\infty} \sum_{\ell=1}^{\infty} z^{k} q^{k \ell}-\sum_{k=1}^{\infty} z^{k}=-\mathcal{L}_{1}(1 / z ; q)-\frac{z}{1-z}
$$

\subsection{Construction de combinaisons linéaires en les $\zeta_{q}(s)$}

Introduisons la fraction rationnelle en $T$ :

$$
R_{n}(T ; q)=(q ; q)_{n}^{A-2 r} q^{-(A-2 r) n / 4} \frac{\left(q^{-r n} T ; q\right)_{r n}\left(q^{n+1} T ; q\right)_{r n}}{(T ; q)_{n+1}^{A}} T^{(A-2 r) n / 2}
$$

avec $n \geqslant 0, A$ pair et $1 \leqslant r \leqslant A / 2$. Définissons également la série :

$$
S_{n}(z ; q)=\sum_{k=1}^{\infty} q^{k} R_{n}\left(q^{k} ; q\right) z^{-k}
$$


qui converge (au moins) pour tout $z$ et tout $q$ tels que $|q|<|z| \leqslant 1$ (c'est ici que l'on utilise la condition $r \leqslant A / 2)$ : en $z=1$, cette série cöncide avec la série $S_{n}(q)$ introduite au paragraphe 3.1. En utilisant la notation classique

$$
{ }_{r} \phi_{s}\left[\begin{array}{l}
a_{1}, \ldots, a_{r} \\
b_{1}, \ldots, b_{s}
\end{array} ; q, z\right]=\sum_{n=0}^{\infty} \frac{\left(a_{1} ; q\right)_{n} \cdots\left(a_{r} ; q\right)_{n}}{(q ; q)_{n}\left(b_{1} ; q\right)_{n} \cdots\left(b_{s} ; q\right)_{n}}\left((-1)^{n} q^{\left(\begin{array}{c}
n \\
2
\end{array}\right)}\right)^{s-r+1} z^{n}
$$

pour la série hypergéométrique basique (voir [10]), on peut écrire $S_{n}(z ; q)$ comme

$$
\begin{aligned}
q^{1+(A n / 4)+(r n / 2)+\left(r A n^{2} / 2\right)-r^{2} n^{2}} \frac{(q ; q)_{n}^{A-2 r}(q ; q)_{r n}\left(q^{(r+1) n+2} ; q\right)_{r n}}{z^{r n+1}\left(q^{r n+1} ; q\right)_{n+1}^{A}} \\
\quad{ }_{A+2} \phi_{A+1}\left[\begin{array}{c}
q^{(2 r+1) n+2}, q^{r n+1}, \ldots, q^{r n+1} \\
q^{(r+1) n+2}, \ldots, q^{(r+1) n+2}
\end{array} ; q, \frac{q^{((A-2 r) n+1) / 2}}{z}\right] .
\end{aligned}
$$

Décomposons $R_{n}(T ; q)$ en éléments simples :

$$
\begin{aligned}
R_{n}(T ; q)= & q^{-A n(n+1) / 2}(q ; q)_{n}^{A-2 r} q^{-(A-2 r) n / 4} \\
& \times \frac{\left(1-q^{-r n} T\right) \cdots\left(1-q^{-1} T\right) \cdot\left(1-q^{n+1} T\right) \cdots\left(1-q^{(r+1) n} T\right)}{(T-1)^{A} \cdots\left(T-q^{-n}\right)^{A}} T^{(A-2 r) n / 2} \\
= & \sum_{s=1}^{A} \sum_{j=0}^{n} \frac{c_{s, j, n}(q)}{\left(T-q^{-j}\right)^{s}}=\sum_{s=1}^{A} \sum_{j=0}^{n} \frac{d_{s, j, n}(q)}{\left(1-q^{j} T\right)^{s}}
\end{aligned}
$$

avec $d_{s, j, n}(q)=(-1)^{s} q^{j s} c_{s, j, n}(q)$. Il n'y a pas de partie principale car la différence des degrés du numérateur et du dénominateur de $R_{n}(T ; q)$ est $-A-(A-2 r) n / 2$, qui est strictement négative puisque $1 \leqslant r \leqslant A / 2$; de plus, selon la formule usuelle, on a

$$
c_{s, j, n}(q)=\left.\frac{1}{(A-s) !} \frac{\mathrm{d}^{A-s}}{\mathrm{~d} T^{A-s}}\left(R_{n}(T ; q)\left(T-q^{-j}\right)^{A}\right)\right|_{T=q^{-j}} .
$$

On construit alors des combinaisons linéaires en les fonctions $\mathcal{Z}_{s}(z ; q)$ de la façon suivante :

$$
\begin{aligned}
S_{n}(z ; q) & =\sum_{s=1}^{A} \sum_{j=0}^{n} d_{s, j, n}(q) q^{-j} z^{j} \sum_{k=1}^{\infty} \frac{q^{k+j}}{\left(1-q^{k+j}\right)^{s}} z^{-k-j} \\
& =P_{0, n}(z ; q)+\sum_{s=1}^{A} P_{s, n}(z ; q) \mathcal{Z}_{s}(z ; q)
\end{aligned}
$$

avec, pour $s \geqslant 1$,

$$
P_{s, n}(z ; q)=\sum_{j=0}^{n} d_{s, j, n}(q) q^{-j} z^{j} \quad \text { et } \quad P_{0, n}(z ; q)=-\sum_{s=1}^{A} \sum_{j=1}^{n} \sum_{k=1}^{j} d_{s, j, n}(q) q^{k-j} \frac{z^{j-k}}{\left(1-q^{k}\right)^{s}} .
$$

Jusqu'à présent, nous n'avons pas exploité la forme particulière du numérateur de $R_{n}(T ; q)$, ce que nous allons maintenant faire. 
Lemme 3.3. Soit $A$ pair. Pour tout $s \in\{1, \ldots, A\}$, on a la relation de réciprocité :

$$
z^{n} q^{-n} P_{s, n}(1 / z ; 1 / q)=P_{s, n}(z ; q) .
$$

Preuve. On vérifie immédiatement que

$$
R_{n}\left(q^{n} T ; 1 / q\right)=R_{n}(T ; q) .
$$

Par unicité du développement en éléments simples (3.13), cette symétrie de $R_{n}(T ; q)$ se traduit par

$$
d_{s, n-j, n}(q)=d_{s, j, n}(1 / q)
$$

ce qui prouve le lemme.

En utilisant l'identité triviale $\left(1 / q^{\alpha} ; 1 / q\right)_{\beta}=(-1)^{\beta} q^{-\alpha \beta-\beta(\beta-1) / 2}\left(q^{\alpha} ; q\right)_{\beta}$, on montre sans difficulté que

$$
S_{n}(1 / z ; 1 / q)=q^{A n / 2} S_{n}\left(q^{2-A} / z ; q\right)
$$

qui est convergente sur $|q|<|z| \leqslant 1$ : on peut donc considérer simultanément les séries $S_{n}(z ; q)$ et $S_{n}(1 / z ; 1 / q)$. Cela va nous permettre de construire une nouvelle combinaison linéaire réalisant la dichotomie attendue entre les valeurs de la fonction $\zeta_{q}(s)$ aux entiers $s$ pairs et impairs.

Lemme 3.4. Soit $A$ pair et $\epsilon \in\{0,1\}$. Il existe des fractions rationnelles $P_{s, n}^{[\epsilon]}(q)$ de $\mathbb{Q}(q)$, avec $s \in\{0, \ldots, A\}$, telles que

$$
S_{n}^{[\epsilon]}(q)=S_{n}(q)+(-1)^{\epsilon} q^{-n} S_{n}(1 / q)=P_{0, n}^{[\epsilon]}(q)+\sum_{\substack{s=2 \\ s \equiv \epsilon(\bmod 2)}}^{A} P_{s, n}^{[\epsilon]}(q) \zeta_{q}(s) .
$$

On explicitera les $P_{s, n}^{[\epsilon]}(q)$ au cours de la démonstration.

Preuve. On se concentre sur le cas $\epsilon=1$, le cas $\epsilon=0$ étant similaire. Compte-tenu de la relation de réciprocité mise en évidence au Lemme 3.3, on a

$$
\begin{aligned}
S_{n}(z ; q) & -q^{-n} z^{n} S_{n}(1 / z ; 1 / q) \\
& =P_{0, n}(z ; q)-q^{-n} z^{n} P_{0, n}(1 / z ; 1 / q)+\sum_{s=1}^{A} P_{s, n}(z ; q)\left(\mathcal{Z}_{s}(z ; q)-\mathcal{Z}_{s}(1 / z ; 1 / q)\right) .
\end{aligned}
$$

Les séries $S_{n}(z ; q)$ et $q^{-n} z^{n} S_{n}(1 / z ; 1 / q)$ sont convergentes en $z=1$, ainsi que les fonctions $\mathcal{Z}_{s}(z ; q)-\mathcal{Z}_{s}(1 / z ; 1 / q)$ pour $s \geqslant 2$. Le seul terme potentiellement divergent est $\mathcal{Z}_{1}(z ; q)-\mathcal{Z}_{1}(1 / z ; 1 / q)$ : pour contrer cette divergence, le polynôme (en la variable $z$ ) $P_{1, n}(z ; q)$ s'annule nécessairement en $z=1$. Or en utilisant le point (iii) du Lemme 3.2, on a

$$
\begin{aligned}
& \lim _{z \rightarrow 1^{-}} P_{1, n}(z ; q)\left(\mathcal{Z}_{1}(z ; q)-\mathcal{Z}_{1}(1 / z ; 1 / q)\right) \\
& \quad=\lim _{z \rightarrow 1^{-}} P_{1, n}(z ; q)\left(\mathcal{L}_{1}(z ; q)+\mathcal{L}_{1}(1 / z ; q)\right)+\lim _{z \rightarrow 1^{-}} \frac{z P_{1, n}(z ; q)}{1-z}=-\frac{\partial P_{1, n}}{\partial z}(1 ; q)
\end{aligned}
$$


puisque $\mathcal{L}_{1}(1 ; q)$ est convergente. Par ailleurs, le point (i) du même Lemme 3.2 montre que pour $s \geqslant 2$,

$$
\mathcal{Z}_{s}(q)-\mathcal{Z}_{s}(1 / q)=\sum_{\substack{j=3 \\ j \text { impair }}}^{s} \alpha_{s, j} \zeta_{q}(j)
$$

où les coefficients $\alpha_{s, j}$ sont des rationnels indépendants de $q$ et dont un dénominateur commun est $(s-1)$ ! (ce fait nous servira au cours de la démonstration du Lemme 3.7 ; en fait, $\alpha_{s, j}=2 c(s-1, j-1) /(s-1)$ !, où $c(s-1, j-1)$ est un nombre de Stirling de première espèce sans signe). Donc

$$
S_{n}^{[1]}(q)=P_{0, n}(1 ; q)-q^{-n} P_{0, n}(1 ; 1 / q)-\frac{\partial P_{1, n}}{\partial z}(1 ; q)+\sum_{s=3}^{A} \sum_{\substack{j=3 \\ j \text { impair }}}^{s} \alpha_{s, j} P_{s, n}(1 ; q) \zeta_{q}(j) .
$$

Il suffit donc de poser

$$
P_{0, n}^{[1]}(q)=P_{0, n}(1 ; q)-q^{-n} P_{0, n}(1 ; 1 / q)-\frac{\partial P_{1, n}}{\partial z}(1 ; q)
$$

et, pour $s$ impair de $\{3, \ldots, A-1\}$,

$$
P_{s, n}^{[1]}(q)=\sum_{k=s}^{A} \alpha_{k, s} P_{k, n}(1 ; q) .
$$

Dans le cas $\epsilon=0$, on trouve

$$
P_{0, n}^{[0]}(q)=P_{0, n}(1 ; q)+q^{-n} P_{0, n}(1 ; 1 / q)+\frac{\partial P_{1, n}}{\partial z}(1 ; q)
$$

et, pour $s$ pair de $\{2, \ldots, A\}$,

$$
P_{s, n}^{[0]}(q)=\sum_{k=s}^{A} \alpha_{k, s} P_{k, n}(1 ; q) .
$$

Lorsque l'on essaye de relier la série $S_{n}^{[\epsilon]}(q)$ à la notation classique (3.12), on obtient

$$
\begin{aligned}
& S_{n}^{[\epsilon]}(q)=\sum_{k=1}^{\infty} q^{k} R_{n}\left(q^{k} ; q\right)\left(1+(-1)^{\epsilon} q^{(A / 2-1)(n+2 k)}\right) \\
& =q^{1+(A n / 4)+(r n / 2)+\left(r A n^{2} / 2\right)-r^{2} n^{2}} \frac{(q ; q)_{n}^{A-2 r}(q ; q)_{r n}\left(q^{(r+1) n+2} ; q\right)_{r n}}{\left(q^{r n+1} ; q\right)_{n+1}^{A}} \\
& \times \sum_{k=0}^{\infty}\left(1+(-1)^{\epsilon} q^{(A / 2-1)((2 r+1) n+2 k+2)}\right) \\
& \times \frac{\left(q^{(2 r+1) n+2} ; q\right)_{k}\left(q^{r n+1} ; q\right)_{k}^{A+1}}{\left(q^{(r+1) n+2} ; q\right)_{k}^{A+1}}\left(q^{((A-2 r) n+1) / 2}\right)^{k} .
\end{aligned}
$$


À cause du facteur $\left(1+(-1)^{\epsilon} q^{(A / 2-1)((2 r+1) n+2 k+2)}\right)$, il n'y a pas d'écriture élégante de cette somme en notation hypergéométrique basique car il faudrait pour cela utiliser des racines $(A-2)$ ièmes de l'unité. La somme est cependant une série bien équilibrée ('well-poised'; voir $[\mathbf{1 0}, \S 2.1]$ ), et même très bien équilibrée ('very well-poised') si $\epsilon$ est impair. Remarquons que la série alternative $\tilde{S}_{n}(q)$ donnée par (3.3) est une série très bien équilibrée.

\subsection{Estimation asymptotique de $S_{n}^{[\epsilon]}(q)$}

Dans ce paragraphe on démontre le point (B) du paragraphe 3.1 .

Lemme 3.5. Soit $A \geqslant 2 r$. Pour $\epsilon \in\{0,1\}$ et $q$ tel que $|q|<1$, on a

$$
\lim _{n \rightarrow+\infty} \frac{1}{n^{2}} \log \left|S_{n}^{[\epsilon]}(q)\right|=-\frac{1}{2} r(A-2 r) \log |1 / q| .
$$

Preuve. Fixons $A, r$ et $q$. Notons $\rho_{k}(q)=q^{k} R_{n}\left(q^{k} ; q\right)$ le sommande de la série $S_{n}(q)$ : clairement, $\rho_{k}(q)=0$ pour $k \in\{1, \ldots, r n\}$ et $\rho_{k}(q) \neq 0$ pour tout $k \geqslant r n+1$. Il est immédiat que pour $k \geqslant r n+1$,

$$
\frac{\rho_{k+1}(q)}{\rho_{k}(q)}=q^{(A-2 r) n / 2+1} \frac{1-q^{1+k+n+r n}}{1-q^{k-r n}}\left(\frac{1-q^{k}}{1-q^{k+n+1}}\right)^{A+1} .
$$

Donc, puisque $|q|<1$ et $k \geqslant r n+1$,

$$
\left|\frac{\rho_{k+1}(q)}{\rho_{k}(q)}\right| \leqslant|q|^{(A-2 r) n / 2}\left(\frac{1+|q|}{1-|q|}\right)^{A+2}<\frac{1}{3},
$$

pourvu que $n \gg 0$ (ce qui dépend de $A, r$ et $q$ mais pas de $k$ ). À cette condition, on a d'un côté

$$
\left|S_{n}(q)\right|=\left|\rho_{r n+1}(q)\right|\left|\sum_{k=r n+1}^{\infty} \frac{\rho_{k}(q)}{\rho_{r n+1}(q)}\right|<\left|\rho_{r n+1}(q)\right| \sum_{k=0}^{\infty} 3^{-k}=\frac{3}{2}\left|\rho_{r n+1}(q)\right|,
$$

et d'un autre côté,

$$
\left|S_{n}(q)\right| \geqslant\left|\rho_{r n+1}(q)\right|\left(1-\sum_{k=r n+2}^{\infty}\left|\frac{\rho_{k}(q)}{\rho_{r n+1}(q)}\right|\right) \geqslant \frac{1}{2}\left|\rho_{r n+1}(q)\right| .
$$

Comme

$$
\rho_{r n+1}(q)=(q ; q)_{n}^{A-2 r} q^{-(A-2 r) n / 4} \frac{(q ; q)_{r n}\left(q^{(r+1) n+2} ; q\right)_{r n}}{\left(q^{r n+1} ; q\right)_{n+1}^{A}} q^{(A-2 r)(r n+1) n / 2}
$$

et

$$
\lim _{n \rightarrow+\infty} \frac{1}{n^{2}} \log \left|(q ; q)_{n}^{A-2 r} q^{-(A-2 r) n / 4} \frac{(q ; q)_{r n}\left(q^{(r+1) n+2} ; q\right)_{r n}}{\left(q^{r n+1} ; q\right)_{n+1}^{A}}\right|=0
$$


on obtient

$$
\lim _{n \rightarrow+\infty} \frac{1}{n^{2}} \log \left|S_{n}(q)\right|=\lim _{n \rightarrow+\infty} \frac{1}{n^{2}} \log \left|q^{(A-2 r)(r n+1) n / 2}\right|=-\frac{1}{2} r(A-2 r) \log |1 / q| .
$$

La série qui nous intéresse à proprement parler est $S_{n}^{[\epsilon]}(q)$, que l'on peut écrire, selon (3.16), sous la forme

$$
S_{n}^{[\epsilon]}(q)=\sum_{k=r n+1}^{\infty}\left(1+(-1)^{\epsilon} q^{(A / 2-1)(n+2 k)}\right) \rho_{k}(q) .
$$

Le facteur $1+(-1)^{\epsilon} q^{(A / 2-1)(n+2 k)}$ n'a aucune influence asymptotique par rapport à $\rho_{k}(q)$ quand $n \rightarrow+\infty$ et est toujours non nul puisque $|q|<1$. En procédant exactement comme ci-dessus, on montre alors que le terme d'indice $k=r n+1$ domine les suivants et que

$$
\lim _{n \rightarrow+\infty} \frac{1}{n^{2}} \log \left|S_{n}^{[\epsilon]}(q)\right|=-\frac{1}{2} r(A-2 r) \log |1 / q| .
$$

\subsection{Estimation asymptotique de $P_{s, n}^{[\epsilon]}(q)$}

Dans ce paragraphe on démontre le point $(\mathrm{C})$ du paragraphe 3.1.

Lemme 3.6. Pour tous $\epsilon \in\{0,1\}, s \in\{0, \ldots, A\}$ et $q$ tel que $|q|<1$, on a

$$
\limsup _{n \rightarrow+\infty} \frac{1}{n^{2}} \log \left|P_{s, n}^{[\epsilon]}(q)\right| \leqslant \frac{1}{8}\left(A+4 r^{2}\right) \log |1 / q| .
$$

Preuve. Étant données les expressions des diverses fonctions $P_{s, n}^{[\epsilon]}(q)$ apparues au court de la démonstration du Lemme 3.4, il suffit de montrer que l'estimation attendue est déjà valide pour les coefficients $d_{s, j, n}(q)=(-1)^{s} q^{j s} c_{s, j, n}(q)$, uniformément en $j$ et $s$. Fixons $q$ tel que $|q|<1$ et $j \in\{0, \ldots, n\}$ et soit $\eta>0$ assez petit (on peut choisir $\eta=(1-|q|) / 2)$ : par la formule de Cauchy appliquée à (3.14), on obtient

$$
c_{s, j, n}(q)=\frac{1}{2 \pi \mathrm{i}} \int_{\mathcal{C}} R_{n}(T ; q)\left(T-q^{-j}\right)^{s-1} \mathrm{~d} T,
$$

où $\mathcal{C}$ désigne le cercle de centre $q^{-j}$ et de rayon $\eta|q|^{-j}$. En remplaçant $T$ par $T q^{-j}$, cela équivaut à

$$
d_{s, j, n}(q)=-\frac{1}{2 \pi \mathrm{i}} \int_{\mathcal{C}^{\prime}} R_{n}\left(T q^{-j} ; q\right)(1-T)^{s-1} \mathrm{~d} T,
$$

où $\mathcal{C}^{\prime}$ désigne le cercle de centre 1 et de rayon $\eta$. Il s'agit donc de majorer la fraction

$$
\begin{aligned}
R_{n}\left(T q^{-j} ; q\right)(1-T)^{s-1} & \\
= & q^{-(A-2 r)(2 j+1) n / 4}(q ; q)_{n}^{A-2 r} \frac{\left(q^{-r n-j} T ; q\right)_{r n}\left(q^{n-j+1} T ; q\right)_{r n}(1-T)^{s-1}}{\left(T q^{-j} ; q\right)_{n+1}^{A}} T^{(A-2 r) n / 2} \\
= & (-1)^{A j+n r} q^{A\left(\begin{array}{c}
j+1 \\
2
\end{array}\right)-(A n / 4)(2 j+1)-\left(r^{2} n^{2} / 2\right)}(q ; q)_{n}^{A-2 r} \\
& \times \frac{\left(q^{j+1} / T ; q\right)_{r n}\left(q^{n-j+1} T ; q\right)_{r n}(1-T)^{s-A-1}}{(q / T ; q)_{j}^{A}(q T ; q)_{n-j}^{A}} T^{A(n-2 j) / 2}
\end{aligned}
$$


$\operatorname{sur} \mathcal{C}^{\prime}$. On vérifie que pour tous nombres entiers positifs $a, b$, avec $a>0$, et tout $T \in \mathcal{C}$, on a

$$
0<(|q|(1+\eta) ;|q|)_{\infty} \leqslant\left|\left(q^{a} T ; q\right)_{b}\right| \leqslant(-(1+\eta) ;|q|)_{\infty}
$$

et

$$
0<(|q| /(1-\eta) ;|q|)_{\infty} \leqslant\left|\left(q^{a} / T ; q\right)_{b}\right| \leqslant(-1 /(1-\eta) ;|q|)_{\infty} .
$$

Les bornes inférieures sont effectivement $>0$ parce que les valeurs $|q|(1+\eta)$ et $|q| /(1-\eta)$ sont $<1$. On a également

$$
\left|(q ; q)_{n}\right| \leqslant(-|q| ;|q|)_{\infty} \quad \text { et }\left|T^{(n-2 j) A / 2}\right| \leqslant(\max \{1+\eta, 1 /(1-\eta)\})^{A n / 2} .
$$

Finalement, pour l'exposant de $q$ dans le membre final de (3.17), on a

$$
A\left(\begin{array}{c}
j+1 \\
2
\end{array}\right)-\frac{A n}{4}(2 j+1)-\frac{r^{2} n^{2}}{2} \geqslant-\frac{A}{8}-\frac{A n^{2}}{8}-\frac{r^{2} n^{2}}{2}
$$

pour tout $j \in\{0,1, \cdots, n\}$. On en déduit que

$$
\left|d_{s, j, n}(q)\right| \leqslant C_{0}|q|^{-\left(A+4 r^{2}\right) n^{2} / 8},
$$

où $C_{0}$ dépend de $A, \eta, n, q$ et $r$ mais ni de $j$ ni de $s$, et vérifie $C_{0}^{1 / n^{2}} \rightarrow 1$ quand $n \rightarrow+\infty$.

Remarque. Nous pensons qu'en fait

$$
\lim _{n \rightarrow+\infty} \frac{1}{n^{2}} \log \left|P_{s, n}^{[\epsilon]}(q)\right|=\frac{1}{8}\left(A+4 r^{2}\right) \log |1 / q|,
$$

ce qui est supporté par des calculs numériques effectués sur ordinateur, ainsi que par des arguments heuristiques. Si cela est vrai, on pourra alors étendre les Théorèmes 1.2 et 1.3 aux valeurs algébriques de $q$ en utilisant un critère d'indépendance linéaire sur les corps de nombres (voir $[\mathbf{4}, \mathbf{1 2}, \mathbf{2 7}]$ ).

\subsection{Estimation arithmétique de $P_{s, n}^{[\epsilon]}(q)$}

Dans ce paragraphe on démontre le point (D) du paragraphe 3.1. Laissons temporairement $q$ être tel que $|q| \neq 1$. Rappelons que l'on définit les coefficients $q$-binomiaux par

$$
\left[\begin{array}{c}
n \\
k
\end{array}\right]_{q}=\frac{(q ; q)_{n}}{(q ; q)_{k}(q ; q)_{n-k}}
$$

et qu'ils appartiennent à $\mathbb{Z}[q]$. Pour tout $n \geqslant 0$, le polynôme unitaire $d_{n}(q)$ de plus petit degré en $q$ et tel que

$$
d_{n}(q) \frac{1}{1-q}, d_{n}(q) \frac{1}{1-q^{2}}, \ldots, d_{n}(q) \frac{1}{1-q^{n}} \in \mathbb{Z}[q],
$$


est donné par $d_{n}(q)=\prod_{\ell=1}^{n} \Phi_{\ell}(q)$, où $\Phi_{\ell}(q)=\prod_{k=1,(k, \ell)=1}^{\ell}\left(q-\mathrm{e}^{2 \mathrm{i} \pi k / \ell}\right)$ est le $\ell$ ième polynôme cyclotomique. Il satisfait l'estimation suivante pour tout $q$ tel que $|q|>1$ (voir $[\mathbf{8}, \S 2]$ et $[\mathbf{2 8}$, Lemma 2]) :

$$
\lim _{n \rightarrow+\infty} \frac{1}{n^{2}} \log \left|d_{n}(q)\right|=\frac{3}{\pi^{2}} \log |q| .
$$

Nous sommes maintenant en position d'expliciter les fractions rationnelles $D_{n}(q)$ qui apparaissent au point (D) du paragraphe 3.1 et d'établir leur comportement asymptotique lorsque $n$ tend vers $+\infty$. Soit

$$
D_{n}(q)=(A-1) ! q^{(A-2 r) n / 4-\left\lceil A(n+1)^{2} / 8\right\rceil-\left(r^{2} n^{2} / 2\right)+(r n / 2)-(A-1) n} d_{n}(1 / q)^{A} .
$$

On a alors le lemme suivant.

Lemme 3.7. Soit $A$ pair.

(i) Pour tous $\epsilon \in\{0,1\}, s \in\{0, \ldots A\}$ et $q$ tel que $|q| \neq 1$, on a

$$
D_{n}(q) P_{s, n}^{[\epsilon]}(q) \in \mathbb{Z}[1 / q] .
$$

(ii) Lorsque $|q|<1$, on a alors

$$
\lim _{n \rightarrow+\infty} \frac{1}{n^{2}} \log \left|D_{n}(q)\right|=\left(\frac{3}{\pi^{2}} A+\frac{A}{8}+\frac{r^{2}}{2}\right) \log |1 / q| .
$$

Preuve. Nous allons d'abord démontrer que pour tous $s \in\{0, \ldots, A\}, j \in\{0, \ldots, n\}$ et $q$ tel que $|q| \neq 1$, on a

$$
q^{(A-2 r) n / 4-\left\lceil A(n+1)^{2} / 8\right\rceil-\left(r^{2} n^{2} / 2\right)+(r n / 2)-n} d_{n}(1 / q)^{A-s} c_{s, j, n}(q) \in \mathbb{Z}[1 / q],
$$

le coefficient $c_{s, j, n}(q)$ étant donné par (3.14). Décomposons tout d'abord $R_{n}(T ; q)$ de la façon suivante :

$$
\begin{gathered}
R_{n}(T ; q)\left(T-q^{-j}\right)^{A}=q^{-(A-2 r) n / 4}\left(q^{-n(n+1) / 2}(q ; q)_{n} \frac{T^{n}\left(T-q^{-j}\right)}{(T-1) \cdots\left(T-q^{-n}\right)}\right)^{(A-2 r) / 2} \\
\times\left(q^{-n(n+1) / 2}(q ; q)_{n} \frac{\left(T-q^{-j}\right)}{(T-1) \cdots\left(T-q^{-n}\right)}\right)^{(A-2 r) / 2} \\
\times \prod_{\ell=1}^{r}\left(q^{-n(n+1) / 2} \frac{\left(q^{-\ell n} T ; q\right)_{n}\left(T-q^{-j}\right)}{(T-1) \cdots\left(T-q^{-n}\right)}\right) \\
\times \prod_{\ell=1}^{r}\left(q^{-n(n+1) / 2} \frac{\left(q^{\ell n+1} T ; q\right)_{n}\left(T-q^{-j}\right)}{(T-1) \cdots\left(T-q^{-n}\right)}\right) .
\end{gathered}
$$

On vérifie en décomposant en éléments simples que l'on a les quatre identités suivantes :

$$
\begin{aligned}
F(T) & =q^{-n(n+1) / 2}(q ; q)_{n} \frac{T^{n}\left(T-q^{-j}\right)}{(T-1) \cdots\left(T-q^{-n}\right)} \\
& =(-1)^{n}(1 / q ; 1 / q)_{n}+\sum_{\substack{i=0 \\
i \neq j}}^{n}(-1)^{n+i} q^{-i(i+1) / 2}\left[\begin{array}{c}
n \\
i
\end{array}\right]_{1 / q} \frac{q^{-i}-q^{-j}}{T-q^{-i}},
\end{aligned}
$$




$$
\begin{aligned}
G(T) & =\frac{q^{-n(n+1) / 2}(q ; q)_{n}\left(T-q^{-j}\right)}{(T-1) \cdots\left(T-q^{-n}\right)} \\
& =\sum_{\substack{i=0 \\
i \neq j}}^{n}(-1)^{n+i} q^{i n-i(i+1) / 2}\left[\begin{array}{c}
n \\
i
\end{array}\right]_{1 / q} \frac{q^{-i}-q^{-j}}{T-q^{-i}}, \\
H_{\ell}(T) & =q^{-n(n+1) / 2} \frac{\left(q^{-\ell n} T ; q\right)_{n}\left(T-q^{-j}\right)}{(T-1) \cdots\left(T-q^{-n}\right)} \\
& =(-1)^{n} q^{-\ell n^{2}-n}+\sum_{\substack{i=0 \\
i \neq j}}^{n}(-1)^{i} q^{-(n-i)^{2} / 2-(n+i) / 2}\left[\begin{array}{c}
n \\
i
\end{array}\right]_{1 / q}\left[\begin{array}{c}
\ell n+i \\
n
\end{array}\right]_{1 / q} \frac{q^{-i}-q^{-j}}{T-q^{-i}}
\end{aligned}
$$

et

$$
\begin{aligned}
I_{\ell}(T) & =q^{-n(n+1) / 2} \frac{\left(q^{\ell n+1} T ; q\right)_{n}\left(T-q^{-j}\right)}{(T-1) \cdots\left(T-q^{-n}\right)} \\
& =(-1)^{n} q^{\ell n^{2}}\left(1+\sum_{\substack{i=0 \\
i \neq j}}^{n}(-1)^{i} q^{-i(i+1) / 2}\left[\begin{array}{c}
n \\
i
\end{array}\right]_{1 / q}\left[\begin{array}{c}
\ell(n+1)-i \\
n
\end{array}\right]_{1 / q} \frac{q^{-i}-q^{-j}}{T-q^{-i}}\right) .
\end{aligned}
$$

Notons

$$
\partial^{\mu}=\frac{1}{\mu !} \frac{\mathrm{d}^{\mu}}{\mathrm{d} T^{\mu}}
$$

Il résulte des formules $(3.20),(3.21),(3.22),(3.23)$, que pour tout entier $\mu \geqslant 0$, tout $j \in\{0, \ldots, n\}$ et tout $\ell \in\{1, \ldots, r\}$, les quantités

$$
d_{n}(1 / q)^{\mu} \partial^{\mu} F\left(q^{-j}\right), \quad d_{n}(1 / q)^{\mu} \partial^{\mu} G\left(q^{-j}\right), \quad d_{n}(1 / q)^{\mu} \partial^{\mu} H_{\ell}\left(q^{-j}\right), \quad d_{n}(1 / q)^{\mu} \partial^{\mu} I_{\ell}\left(q^{-j}\right),
$$

sont des polynômes en $1 / q$, multipliés par une certaine puissance de $q$ et dont les coefficients sont des nombres entiers. Or la formule de Leibniz nous donne

$$
\begin{aligned}
c_{s, j, n}(q)=q^{-(A-2 r) n / 4} & \sum_{\boldsymbol{\mu}}\left(\partial^{\mu_{1}} F\right) \cdots\left(\partial^{\mu_{(A-2 r) / 2}} F\right)\left(\partial^{\mu_{(A-2 r) / 2+1}} G\right) \cdots \\
& \cdots\left(\partial^{\mu_{A-2 r}} G\right)\left(\partial^{\mu_{A-2 r+1}} H_{1}\right) \cdots\left(\partial^{\mu_{A-r}} H_{r}\right)\left(\partial^{\mu_{A-r+1}} I_{1}\right) \cdots\left(\partial^{\mu_{A}} I_{r}\right)
\end{aligned}
$$

(où la sommation est sur tous les multiplets $\boldsymbol{\mu}=\left(\mu_{1}, \ldots, \mu_{A}\right)$ tels que $\mu_{1}+\cdots+\mu_{A}=A-s$, et où l'on a omis l'évaluation en $T=q^{-j}$ pour simplifier). On en déduit que

$$
q^{(A-2 r) n / 4} d_{n}(1 / q)^{A-s} c_{s, j, n}(q)
$$

est un polynôme en $1 / q$, à coefficients entiers et multiplié par une certaine puissance de $q$.

Il nous faut maintenant expliciter cette puissance de $q$, c'est-à-dire déterminer un exposant $e(n)$ le plus grand possible tel que

$$
q^{e(n)} q^{(A-2 r) n / 4} d_{n}(1 / q)^{A-s} c_{s, j, n}(q) \in \mathbb{Z}[1 / q] .
$$


Pour cela, revenons à la définition originelle (3.14) de $c_{s, j, n}(q)$. Posons pour simplifier $R(T)=R_{n}(T ; q)\left(T-q^{-j}\right)^{A}$. En utilisant la formule de Faà di Bruno (voir [13]), on a d'un côté

$$
\begin{aligned}
\partial^{\mu} R(T) & =\partial^{\mu} \exp (\log (R(T))) \\
& =\sum \frac{1}{k_{1} ! k_{2} ! \cdots k_{\mu} !}\left(\frac{\partial^{|\boldsymbol{k}|}}{\partial T^{|\boldsymbol{k}|}} \exp \right)(\log (R(T))) \prod_{i=1}^{\mu}\left(\partial^{i} \log (R(T))\right)^{k_{i}} \\
& =\sum \frac{1}{k_{1} ! k_{2} ! \cdots k_{\mu} !} R(T) \prod_{i=1}^{\mu}\left(\frac{1}{i !} \frac{\partial^{i-1}}{\partial T^{i-1}} r(T)\right)^{k_{i}}
\end{aligned}
$$

où la somme porte sur tous les multiplets $\boldsymbol{k}=\left(k_{1}, k_{2}, \ldots, k_{\mu}\right)$ tels que $k_{1}+2 k_{2}+\cdots+\mu k_{\mu}=$ $\mu$, où $|\boldsymbol{k}|$ désigne la somme $k_{1}+k_{2}+\cdots+k_{\mu}$, et où $r(T)$ est la dérivée logarithmique de $R(T)$

$$
\begin{gathered}
r(T)=\frac{\frac{\partial}{\partial T} R(T)}{R(T)}=\frac{(A-2 r) n}{2} \frac{1}{T}+A \sum_{\substack{i=0 \\
i \neq j}}^{n} \frac{q^{i}}{1-q^{i} T}-\sum_{i=-r n}^{-1} \frac{q^{i}}{1-q^{i} T}-\sum_{i=n+1}^{(r+1) n} \frac{q^{i}}{1-q^{i} T} \\
=\frac{(A-2 r) n}{2} \frac{1}{T}+A \sum_{i=0}^{j-1} \frac{q^{i}}{1-q^{i} T}-A \sum_{i=j+1}^{n} \frac{1 / T}{1-q^{-i} / T} \\
-\sum_{i=-r n}^{-1} \frac{q^{i}}{1-q^{i} T}+\sum_{i=n+1}^{(r+1) n} \frac{1 / T}{1-q^{-i} / T} .
\end{gathered}
$$

D'un autre côté, on a

$$
\begin{aligned}
\left.R(T)\right|_{T=q^{-j}}=(-1)^{r n} q^{-(A-2 r) n / 4-A\left(\begin{array}{c}
j+1 \\
2
\end{array}\right)+(A j n / 2)-(r n / 2)+\left(r^{2} n^{2} / 2\right)} \\
\times \frac{(1 / q ; 1 / q)_{n}^{A-2 r}\left(q^{-j-1} ; 1 / q\right)_{r n}\left(q^{-n+j-1} ; 1 / q\right)_{r n}}{(1 / q ; 1 / q)_{j}^{A}(1 / q ; 1 / q)_{n-j}^{A}} .
\end{aligned}
$$

Comme on sait déjà que

$$
\left.q^{(A-2 r) n / 4} d_{n}(1 / q)^{A-s} \partial^{A-s} R(T)\right|_{T=q^{-j}}=q^{(A-2 r) n / 4} d_{n}(1 / q)^{A-s} c_{s, j, n}(q)
$$

est un polynôme en $1 / q$, multiplié par une puissance de $q$, et comme

$$
-\left(\begin{array}{l}
j \\
2
\end{array}\right)+\frac{j n}{2} \leqslant \frac{1}{8}(n+1)^{2},
$$

la combinaison de (3.24), (3.25) et (3.26) montre que

$$
q^{(A-2 r) n / 4-\left\lceil A(n+1)^{2} / 8\right\rceil-\left(r^{2} n^{2} / 2\right)+(r n / 2)-(A-s) n} d_{n}(1 / q)^{A-s} c_{s, j, n}(q)
$$

est un polynôme en $1 / q$ à coefficients entiers. (Lorsque l'on utilise (3.25), des expressions comme $1 /\left(1-q^{-m}\right)^{M}$, où $m$ est un nombre entier positif, sont interprétées comme séries formelles en $1 / q$.) 
On peut maintenant passer à la démonstration du point (i) proprement dit. De nouveau, on se concentre sur le cas $\epsilon=1$. Rappelons que pour $s \geqslant 2$ pair, on a $P_{s, n}(q)=0$ et que pour $s$ impair de $\{3, \ldots, A-1\}$, on a (voir (3.15))

$$
P_{s, n}^{[1]}(q)=\sum_{k=s}^{A} \alpha_{k, s} P_{k, n}(1 ; q),
$$

avec

$$
P_{k, n}(1 ; q)=\sum_{j=0}^{n} d_{k, j, n}(q) q^{-j}=(-1)^{k} \sum_{j=0}^{n} q^{j(k-1)} c_{k, j, n}(q) .
$$

Il résulte donc de l'étude précédente sur $c_{s, j, n}(q)$ (et du dénominateur commun $(A-1)$ ! aux $\alpha_{s, k}$ ) que pour $s \geqslant 3$ :

$$
(A-1) ! q^{(A-2 r) n / 4-\left\lceil A(n+1)^{2} / 8\right\rceil-\left(r^{2} n^{2} / 2\right)+(r n / 2)-(A-s) n} d_{n}(1 / q)^{A-s} P_{s, n}^{[1]}(q) \in \mathbb{Z}[1 / q] .
$$

Par ailleurs,

avec :

$$
P_{0, n}^{[1]}(q)=P_{0, n}(1 ; q)-q^{-n} P_{0, n}(1 ; 1 / q)-\frac{\partial P_{1, n}}{\partial z}(1 ; q)
$$

$$
P_{0, n}(1 ; q)=-\sum_{s=1}^{A} \sum_{j=1}^{n} \sum_{k=1}^{j} d_{s, j, n}(q) \frac{q^{k-j}}{\left(1-q^{k}\right)^{s}},
$$

qui équivaut à

$$
q^{-n} P_{0, n}(1 ; 1 / q)=-\sum_{s=1}^{A} \sum_{j=0}^{n-1} \sum_{k=1}^{n-j}(-1)^{s} c_{s, j, n}(q) \frac{q^{j(s-1)-k}}{\left(1-q^{-k}\right)^{s}}
$$

(en utilisant la relation $d_{s, j, n}(1 / q)=d_{s, n-j, n}(q)$ ), et

$$
\frac{\partial P_{1, n}}{\partial z}(1 ; q)=-\sum_{j=0}^{n} j \cdot c_{1, j, n}(q) .
$$

On déduit de ces trois expressions que

$$
q^{(A-2 r) n / 4-\left\lceil A(n+1)^{2} / 8\right\rceil-\left(r^{2} n^{2} / 2\right)+(r n / 2)-(A-1) n} d_{n}(1 / q)^{A} P_{0, n}^{[1]}(q) \in \mathbb{Z}[1 / q] .
$$

Le point (ii) est évident compte-tenu de (3.18).

\section{4. q-Analogie avec la fonction zêta de Riemann}

Nous terminons cet article en discutant plus en détail deux aspects du travail présenté précédemment. Dans la première partie de ce paragraphe, nous justifions que nos $\zeta_{q}(s)$ sont des $q$-analogues normalisés des nombres $\zeta(s)$, et que nos séries $S_{n}^{[\epsilon]}(q)$ sont des $q$ analogues des séries utilisées récemment dans les travaux sur la dimension des espaces vectoriels engendrés sur $\mathbb{Q}$ par les valeurs de la fonction zêta aux entiers impairs. Dans la deuxième partie, nous présentons des $q$-analogues des séries, maintenant classiques, de Ball et Beukers-Gutnik-Nesterenko, utilisées dans des démonstrations de l'irrationalité de $\zeta(3)$ : ces $q$-analogues sont fortement liés à notre série $S_{n}^{[1]}(q)$ pour $A=4$ et $r=1$. 


\subsection{Convergence $»$ vers le cas « classique $»$}

Dans ce paragraphe, on discute du rapport entre nos $q$-fonctions et $q$-séries et les fonctions et séries «classiques».

Remarquons tout d'abord que les valeurs $\zeta_{q}(s)$ sont des $q$-analogues des valeurs de la fonction zêta de Riemann en nombres entiers $s$ : en utilisant les nombres de Stirling de seconde espèce $S(N, j)$, définis par

$$
\ell^{N}=\sum_{j=1}^{N} S(N, j) \ell(\ell-1) \cdots(\ell-j+1),
$$

on a

$$
\begin{aligned}
\zeta_{q}(s) & =\sum_{k=1}^{\infty} \sum_{d=1}^{\infty} d^{s-1} q^{k d}=\sum_{k=1}^{\infty} \sum_{d=0}^{\infty}(d+1)^{s-1} q^{k(d+1)} \\
& =\sum_{k=1}^{\infty} \sum_{d=0}^{\infty} \sum_{j=1}^{s-1}(-1)^{s-1-j} S(s-1, j) j !\left(\begin{array}{c}
d+j \\
j
\end{array}\right) q^{k(d+1)} \\
& =\sum_{j=1}^{s-1}(-1)^{s-1-j} S(s-1, j) j ! \sum_{k=1}^{\infty} \frac{q^{k}}{\left(1-q^{k}\right)^{j+1}} .
\end{aligned}
$$

La série

$$
\mathcal{Z}_{j+1}(q)=\sum_{k=1}^{\infty} \frac{q^{k}}{\left(1-q^{k}\right)^{j+1}}
$$

étant clairement un $q$-analogue de $\zeta(j+1)$, la série $\zeta_{q}(s)$ est une combinaison linéaire de $q$-analogues des valeurs de la fonction zêta. On voit alors sur l'expression précédente que

$$
\lim _{q \rightarrow 1}(1-q)^{s} \zeta_{q}(s)=(s-1) ! \zeta(s),
$$

et $\zeta_{q}(s)$ peut donc aussi être considérée comme un $q$-analogue normalisé de $\zeta(s)$ (voir $[\mathbf{1 4}$, Theorem 2] et [31] pour d'autres démonstrations de (4.1)).

De même, en utilisant (3.10), (3.11) et (4.1), on obtient

$$
\lim _{q \rightarrow 1}(1-q)^{s}\left(\mathcal{Z}_{s}(q)+(-1)^{\epsilon} \mathcal{Z}_{s}(1 / q)\right)=2 \zeta(s),
$$

si $\epsilon \equiv s \bmod 2$, respectivement

$$
\lim _{q \rightarrow 1}(1-q)^{s-1}\left(\mathcal{Z}_{s}(q)+(-1)^{\epsilon} \mathcal{Z}_{s}(1 / q)\right)=(s-2) \zeta(s-1),
$$

si $\epsilon \not \equiv s$ mod 2. Par conséquent, notre combinaison linéaire $\mathcal{Z}_{s}(q)+(-1)^{\epsilon} \mathcal{Z}_{s}(1 / q)$ (si importante dans la démonstration du Lemme 3.4) est elle aussi un $q$-analogue normalisé de $\zeta(s)$, respectivement $\zeta(s-1)$. En particulier, la combinaison

$$
\frac{1}{2}\left(\mathcal{Z}_{3}(q)-\mathcal{Z}_{3}(1 / q)\right)=\frac{1}{2} \zeta_{q}(3)=\frac{1}{2} \sum_{k=1}^{\infty} \frac{q^{k}\left(1+q^{k}\right)}{\left(1-q^{k}\right)^{3}}
$$

réapparaîtra au paragraphe suivant. 
Comparons maintenant nos séries $S_{n}^{[\epsilon]}(q)$ avec les séries utilisées pour la démonstration du Théorème 1.1. Si l'on multiplie $S_{n}^{[1]}(q) \operatorname{par}(1-q)^{A-1}$ et fait tendre $q$ vers 1 , on obtient la série

$$
(A-2) \cdot n !^{A-2 r} \sum_{k=1}^{\infty}\left(k+\frac{1}{2} n\right) \frac{(k-r n)_{r n}(k+n+1)_{r n}}{(k)_{n+1}^{A}}
$$

où on a encore utilisé les symboles de Pochhammer (voir la démonstration du Lemme 3.2). Aux termes $k+n / 2$ et $(A-2)$ près, il s'agit de la série utilisée dans $[\mathbf{2 , 2 1}]$ pour démontrer le Théorème 1.1. Au terme $A-2$ près, la série (4.3) est aussi un cas spécial d'une série introduite dans [22, p. 51] (et ensuite généralisée dans $[\mathbf{3 4}, \S 8]$ ).

De façon similaire, si l'on multiplie $S_{n}^{[0]}(q)$ par $(1-q)^{A}$ et fait tendre $q$ vers 1 , on obtient la série

$$
2 \cdot n !^{A-2 r} \sum_{k=1}^{\infty} \frac{(k-r n)_{r n}(k+n+1)_{r n}}{(k)_{n+1}^{A}},
$$

qui est ici exactement celle introduite dans $[\mathbf{2}, \mathbf{2 1}]$.

Confronté à cette analogie frappante entre le «cas $q$ général» et le «cas $q=1$ », on aurait pu espérer analogie entre les minorations des dimensions des espaces vectoriels engendrés par les «q-zêtas », respectivement les «zêtas », ce qui n'est pas le cas (comparer les Théorèmes 1.1 et 1.2) : les méthodes appliquées ne permettent pas de démontrer des minorations analogues.

\subsection{Autour de $\zeta_{q}(3)$}

On connaît actuellement de nombreuses démonstrations de l'irrationalité de $\zeta(3)$ (voir l'article de synthèse [9]), dont plusieurs utilisent des séries hypergéométriques. Deux séries classiques sont la série de Ball (qui est le cas $A=4, r=1$ de la série (4.3); voir l'introduction de [21]),

$$
n !^{2} \sum_{k=1}^{\infty}(2 k+n) \frac{(k-n)_{n}(k+n+1)_{n}}{(k)_{n+1}^{4}},
$$

et la série de Beukers-Gutnik-Nesterenko (voir $[\mathbf{6}, \mathbf{1 1}, \mathbf{1 7}]$ ),

$$
-\sum_{k=1}^{\infty} \frac{\mathrm{d}}{\mathrm{d} k}\left(\frac{(k-n)_{n}^{2}}{(k)_{n+1}^{2}}\right)
$$

qui sont en fait égales pour tout nombre entier positif $n$. Dans les preuves d'irrationalité de $\zeta(3)$, on montre que ces séries sont une combinaison linéaire de 1 et $\zeta(3)$, à coefficients rationnelles satisfaisant certaines propriétés asymptotiques et arithmétiques. Dans ce paragraphe nous présentons des $q$-analogues de ces deux séries, et nous démontrons également que ces $q$-analogues sont une combinaison linéaire de 1 et de $\zeta_{q}(3)$. 
Nous commençons avec la version non terminée de la transformation de Watson due à Bailey (voir [10, (2.10.10); Appendix (III.36)]) :

$$
\begin{gathered}
{ }_{8} \phi_{7}\left[\begin{array}{r}
a, \sqrt{a} q,-\sqrt{a} q, b, c, d, e, f \\
\sqrt{a},-\sqrt{a}, a q / b, a q / c, a q / d, a q / e, a q / f
\end{array} ;, \frac{a^{2} q^{2}}{b c d e f}\right] \\
=\frac{(a q, a q / d e, a q / d f, a q / e f ; q)_{\infty}}{(a q / d, a q / e, a q / f, a q / d e f ; q)_{\infty}}{ }_{4} \phi_{3}\left[\begin{array}{c}
a q / b c, d, e, f \\
a q / b, a q / c, \operatorname{def} / a
\end{array} ; q, q\right] \\
+\frac{\left(a q, a q / b c, d, e, f, a^{2} q^{2} / b d e f, a^{2} q^{2} / c d e f ; q\right)_{\infty}}{\left(a q / b, a q / c, a q / d, a q / e, a q / f, a^{2} q^{2} / b c d e f, d e f / a q ; q\right)_{\infty}} \\
\times{ }_{4} \phi_{3}\left[\begin{array}{c}
a q / \operatorname{de}, a q / d f, a q / e f, a^{2} q^{2} / b c d e f \\
a^{2} q^{2} / b d e f, a^{2} q^{2} / c d e f, a q^{2} / \operatorname{def}
\end{array} ; q, q\right]
\end{gathered}
$$

Dans cette identité, on a utilisé la notation

$$
\left(a_{1}, a_{2}, \ldots, a_{k} ; q\right)_{\infty}:=\left(a_{1} ; q\right)_{\infty}\left(a_{2} ; q\right)_{\infty} \cdots\left(a_{k} ; q\right)_{\infty} .
$$

On spécialise alors $a=\delta^{2} q^{3 n+2}$ et $b=c=d=e=f=\delta q^{n+1}$ dans (4.6), et on fait tendre $\delta$ vers 1 . On obtient ainsi

$$
\begin{aligned}
& \frac{\left(q^{n+1} ; q\right)_{n+1}^{4}}{q^{(n+1)^{2}}(q ; q)_{n}\left(q^{2 n+2} ; q\right)_{n+1}} \sum_{k=1}^{\infty} \frac{\left(1-q^{2 k+n}\right)\left(q^{k-n} ; q\right)_{n}\left(q^{1+k+n} ; q\right)_{n}}{\left(q^{k} ; q\right)_{n+1}^{4}} q^{k(n+1)} \\
& =\lim _{\delta \rightarrow 1}\left(\frac{1}{q^{n+1}(q ; q)_{n}} \frac{\left(\delta^{2} q^{3+3 n}, q^{n+1}, q^{n+1}, q^{n+1}, \delta q^{n+1}, \delta q^{n+1}, \delta q^{n+1} ; q\right)_{\infty}}{\left(\delta q^{2 n+2}, \delta q^{2 n+2}, \delta q^{2 n+2}, \delta q^{2 n+2}, \delta q^{2 n+2}, \delta q, q / \delta ; q\right)_{\infty}}\right. \\
& \left.\quad \times \frac{1}{\delta-1}\left(\sum_{k=1}^{\infty} \frac{\left(q^{k-n} ; q\right)_{n}\left(\delta q^{k-n} ; q\right)_{n}}{\left(\delta q^{k} ; q\right)_{n+1}^{2}}\left(\delta q^{k}\right)-\sum_{k=1}^{\infty} \frac{\left(q^{k-n} ; q\right)_{n}\left(q^{k-n} / \delta ; q\right)_{n}}{\left(q^{k} ; q\right)_{n+1}^{2}} q^{k}\right)\right)
\end{aligned}
$$

soit, en utilisant le Théorème de l'Hôpital,

$$
\begin{aligned}
\frac{\left(q^{n+1} ; q\right)_{n+1}^{4}}{q^{(n+1)^{2}}(q ; q)_{n}\left(q^{2 n+2} ; q\right)_{n+1}} \sum_{k=1}^{\infty} & \frac{\left(1-q^{2 k+n}\right)\left(q^{k-n} ; q\right)_{n}\left(q^{1+k+n} ; q\right)_{n}}{\left(q^{k} ; q\right)_{n+1}^{4}} q^{k(n+1)} \\
& =\left.\frac{\left(q^{n+1} ; q\right)_{n}^{4}}{q^{n+1}(q ; q)_{n}^{3}\left(q^{2 n+2} ; q\right)_{n+1}} \sum_{k=1}^{\infty} \frac{\mathrm{d}}{\mathrm{d} \delta}\left(\frac{\left(\delta q^{k-n} ; q\right)_{n}^{2}}{\left(\delta q^{k} ; q\right)_{n+1}^{2}}\left(\delta q^{k}\right)\right)\right|_{\delta=1}
\end{aligned}
$$

Ceci équivaut évidemment à

$$
(q ; q)_{n}^{2} \sum_{k=1}^{\infty} \frac{\left(1-q^{2 k+n}\right)\left(q^{k-n} ; q\right)_{n}\left(q^{1+k+n} ; q\right)_{n}}{\left(q^{k} ; q\right)_{n+1}^{4}} q^{k(n+1)}=\frac{q^{n(n+1)}}{\log q} \sum_{k=1}^{\infty} \frac{\mathrm{d}}{\mathrm{d} k}\left(\frac{\left(q^{k-n} ; q\right)_{n}^{2}}{\left(q^{k} ; q\right)_{n+1}^{2}} q^{k}\right)
$$

dont le membre de gauche est un $q$-analogue parfait de la série (4.4) de Ball et le membre de droite est un $q$-analogue parfait de la série (4.5) de Beukers-Gutnik-Nesterenko. Plus précisément, si l'on multiplie les deux membres de $(4.7)$ par $(1-q)^{3}$ et que l'on fait tendre $q$ vers 1 , on retrouve l'égalité $(4.4)=(4.5)$. Il est également notable que, à un facteur 
$q^{-n / 2}$ près, le membre de gauche est exactement la série $S_{n}^{[1]}(q)=S_{n}(q)-q^{-n} S_{n}(1 / q)$ étudiée au paragraphe 3 , avec $A=4$ et $r=1$.

Poursuivons l'analogie un peu plus loin en utilisant l'identité intégrale de Agarwal (voir $[\mathbf{1 0},(4.6 .5)])$,

$$
\begin{aligned}
& { }_{8} \phi_{7}\left[\begin{array}{c}
q^{a}, q^{1+a / 2},-q^{1+a / 2}, q^{b}, q^{c}, q^{d}, q^{e}, q^{f} \\
\left.q^{a / 2},-q^{a / 2}, q^{1+a-b}, q^{1+a-c}, q^{1+a-d}, q^{1+a-e}, q^{1+a-f} ; q, q^{2+2 a-b-c-d-e-f}\right]
\end{array}\right] \\
& =\sin \pi(b+c+d-a) \\
& \times \frac{\left(q^{1+a}, q^{b}, q^{c}, q^{d}, q^{1+a-b-c}, q^{1+a-b-d}, q^{1+a-c-d}, q^{1+a-e-f} ; q\right)_{\infty}}{\left(q, q^{b+c+d-a}, q^{1+a-b-c-d}, q^{1+a-b}, q^{1+a-c}, q^{1+a-d}, q^{1+a-e}, q^{1+a-f} ; q\right)_{\infty}} \\
& \times \frac{1}{2 \pi \mathrm{i}} \int_{-\mathrm{i} \infty}^{\mathrm{i} \infty} \frac{\left(q^{1+s}, q^{1+b-e+s}, q^{1+a-f+s}, q^{b+c+d-a+s} ; q\right)_{\infty}}{\left(q^{b+s}, q^{c+s}, q^{d+s}, q^{1+a-e-f+s} ; q\right)_{\infty}} \\
& \times \frac{\pi q^{s} \mathrm{~d} s}{\sin \pi s \sin \pi(b+c+d-a+s)},
\end{aligned}
$$

où la courbe d'intégration est déformée autour de l'origine de sorte que celle-ci soit à droite de la courbe. Cette identité est valable à condition que

$$
\operatorname{Re}(s \log q-\log (\sin \pi s \sin \pi(b+c+d-a+s)))<0,
$$

et en la spécialisant en $a=3 n+2$ et $b=c=d=e=f=n+1$, on peut donner une représentation intégrale des séries (4.7). Il s'agit en fait d'un cas limite puisqu'apparait le quotient $(\sin \pi(b+c+d-a)) /\left(q^{1+a-b-c-d} ; q\right)_{\infty}$ :

$$
\begin{aligned}
\lim _{\substack{a \rightarrow 3 n+2 \\
b, c, d \rightarrow n+1}} \frac{\sin \pi(b+c+d-a)}{\left(q^{1+a-b-c-d} ; q\right)_{\infty}} & =\lim _{a \rightarrow 3 n+2} \frac{\sin \pi(3 n+3-a)}{\left(q^{a-3 n-2} ; q\right)_{\infty}} \\
& =\lim _{x \rightarrow 0} \frac{\sin (\pi(1-x))}{\left(1-q^{x}\right)\left(q^{1+x} ; q\right)_{\infty}}=-\frac{\pi}{(q ; q)_{\infty} \log q} .
\end{aligned}
$$

On obtient ainsi :

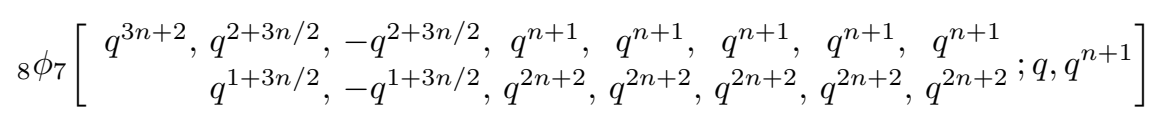

$$
\begin{aligned}
& =\frac{\left(q^{n+1} ; q\right)_{n+1}^{4}}{(q ; q)_{n}^{3}\left(q^{2 n+2} ; q\right)_{n+1}} \frac{1}{\log q} \frac{1}{2 \pi \mathrm{i}} \\
& \times \int_{-\mathrm{i} \infty}^{\mathrm{i} \infty} \frac{\left(q^{1+s}, q^{1+s}, q^{2+2 n+s}, q^{2+2 n+s} ; q\right)_{\infty}}{\left(q^{1+n+s}, q^{1+n+s}, q^{1+n+s}, q^{1+n+s} ; q\right)_{\infty}}\left(\frac{\pi}{\sin \pi s}\right)^{2} q^{s} \mathrm{~d} s .
\end{aligned}
$$

Par conséquent les séries (4.7) s'expriment comme

$$
\frac{q^{(n+1)^{2}}}{\log q} \cdot \frac{1}{2 \pi \mathrm{i}} \int_{-\mathrm{i} \infty}^{\mathrm{i} \infty} \frac{\left(q^{1+s}, q^{1+s}, q^{2+2 n+s}, q^{2+2 n+s} ; q\right)_{\infty}}{\left(q^{1+n+s}, q^{1+n+s}, q^{1+n+s}, q^{1+n+s} ; q\right)_{\infty}}\left(\frac{\pi}{\sin \pi s}\right)^{2} q^{s} \mathrm{~d} s .
$$

Remarquons que l'égalité de (4.8) avec le membre de droite de (4.7) peut être vue directement en poussant la courbe d'intégration à droite et en appliquant le théorème des 
résidus. On obtient ainsi un $q$-analogue parfait d'une autre identité classique (voir [17]) :

$$
-\sum_{k=1}^{\infty} \frac{\mathrm{d}}{\mathrm{d} k}\left(\frac{(k-n)_{n}^{2}}{(k)_{n+1}^{2}}\right)=\frac{1}{2 \mathrm{i} \pi} \int_{-\mathrm{i} \infty}^{\mathrm{i} \infty} \frac{\Gamma(1+s)^{2} \Gamma(2+2 n+s)^{2}}{\Gamma(1+n+s)^{4}}\left(\frac{\pi}{\sin \pi s}\right)^{2} \mathrm{~d} s .
$$

Montrons maintenant que ces deux séries basiques peuvent s'écrire comme combinaison linéaire de 1 et de $\zeta_{q}(3)$. Notons $S_{n}(q)$ la série à droite de $(4.7)$, et

$$
R_{n}(T ; q)=\frac{\left(q^{-n} T ; q\right)_{n}^{2}}{(T ; q)_{n+1}^{2}}
$$

(sans risque de confusion avec les notations utilisées au paragraphe 3) de sorte que

$$
S_{n}(q)=\sum_{k=1}^{\infty} \frac{\mathrm{d}}{\mathrm{d} k}\left(q^{k} R_{n}\left(q^{k} ; q\right)\right) .
$$

Décomposons $R_{n}(T ; q)$ en éléments simples :

$$
R_{n}(T ; q)=\sum_{j=0}^{n}\left(\frac{a_{j, n}(q)}{\left(1-q^{j} T\right)^{2}}+\frac{b_{j, n}(q)}{1-q^{j} T}\right)
$$

d'où

$$
S_{n}(q)=\sum_{j=0}^{n} a_{j, n}(q) q^{-j} \sum_{k=1}^{\infty} \frac{\mathrm{d}}{\mathrm{d} k}\left(\frac{q^{k+j}}{\left(1-q^{k+j}\right)^{2}}\right)+\sum_{j=0}^{n} b_{j, n}(q) q^{-j} \sum_{k=1}^{\infty} \frac{\mathrm{d}}{\mathrm{d} k}\left(\frac{q^{k+j}}{1-q^{k+j}}\right) .
$$

On a donc

$$
\begin{aligned}
S_{n}(q)=\left(\sum_{j=0}^{n} a_{j, n}(q) q^{-j}\right) \sum_{k=1}^{\infty} \frac{\mathrm{d}}{\mathrm{d} k}\left(\frac{q^{k}}{\left(1-q^{k}\right)^{2}}\right)+\left(\sum_{j=0}^{n} b_{j, n}(q) q^{-j}\right) \sum_{k=1}^{\infty} \frac{\mathrm{d}}{\mathrm{d} k}\left(\frac{q^{k}}{1-q^{k}}\right) \\
-\sum_{j=1}^{n} a_{j, n}(q) q^{-j} \sum_{k=1}^{j} \frac{\mathrm{d}}{\mathrm{d} k}\left(\frac{q^{k}}{\left(1-q^{k}\right)^{2}}\right)-\sum_{j=1}^{n} b_{j, n}(q) q^{-j} \sum_{k=1}^{j} \frac{\mathrm{d}}{\mathrm{d} k}\left(\frac{q^{k}}{1-q^{k}}\right) .
\end{aligned}
$$

Remarquons que

$$
\sum_{j=0}^{n} b_{j, n}(q) q^{-j}=-\sum_{j=0}^{n} \operatorname{Res}\left(R_{n}(T ; q)\right)_{T=q^{-j}}=\operatorname{Res}\left(R_{n}(T ; q)\right)_{T=\infty}=0
$$

et que l'on a

$$
\sum_{k=1}^{\infty} \frac{\mathrm{d}}{\mathrm{d} k}\left(\frac{q^{k}}{\left(1-q^{k}\right)^{2}}\right)=(\log q) \sum_{k=1}^{\infty} \frac{q^{k}\left(1+q^{k}\right)}{\left(1-q^{k}\right)^{3}}=(\log q) \zeta_{q}(3) .
$$

Finalement, en posant

$$
A_{n}(q)=\sum_{j=0}^{n} a_{j, n}(q) q^{-j}
$$


et

$$
B_{n}(q)=\sum_{j=1}^{n} \sum_{k=1}^{j} a_{j, n}(q) \frac{q^{k-j}\left(1+q^{k}\right)}{\left(1-q^{k}\right)^{3}}+\sum_{j=1}^{n} \sum_{k=1}^{j} b_{j, n}(q) \frac{q^{k-j}}{\left(1-q^{k}\right)^{2}},
$$

on a donc

$$
S_{n}(q)=(\log q)\left(A_{n}(q) \zeta_{q}(3)-B_{n}(q)\right)
$$

c'est-à-dire

$$
\frac{1}{\log q} \sum_{k=1}^{\infty} \frac{\mathrm{d}}{\mathrm{d} k}\left(\frac{\left(q^{k-n} ; q\right)_{n}^{2}}{\left(q^{k} ; q\right)_{n+1}^{2}} q^{k}\right)=A_{n}(q) \zeta_{q}(3)-B_{n}(q) .
$$

Avec la technique exposée au paragraphe 3.6 , on montre qu'il existe $\bar{D}_{n}(q) \in \mathbb{Q}(q)$ tel que $\bar{D}_{n}(q) A_{n}(q)$ et $\bar{D}_{n}(q) B_{n}(q)$ soient dans $\mathbb{Z}[1 / q]$ et

$$
\lim _{n \rightarrow+\infty} \frac{1}{n^{2}} \log \left|\bar{D}_{n}(q)\right|=\frac{9}{\pi^{2}}
$$

Malheureusement,

$$
\lim _{n \rightarrow+\infty} \frac{1}{n^{2}} \log \left|S_{n}(q)\right|=0
$$

et on ne peut donc montrer l'irrationalité de $\zeta_{q}(3)$ pour aucun $q \neq \pm 1$ tel que $1 / q \in \mathbb{Z}$.

Remarque. Pour finir, notons que, compte-tenu de (4.7), on aurait évidemment pu utiliser le Lemme 3.4 avec $\epsilon=1, A=4$ et $r=1$ pour obtenir (4.9) plus rapidement, mais au prix d'un $\bar{D}_{n}(q)$ moins bon : cela suggère une probable « conjecture des dénominateurs », comparable à celles énoncées dans le cas classique dans [22] et [34]. Plus précisément et comme noté dans la Remarque (2) au paragraphe 3.1, on peut démontrer les propriétés (A)-(D) du paragraphe 3.1 pour la série alternative $\tilde{S}_{n}(q)$, en lieu et place de $S_{n}^{[\epsilon]}(q)$. Il semble alors que l'on peut dans ce cas remplacer le « dénominateur » $D_{n}(q)$ par un $\tilde{D}_{n}(q)$, défini comme $D_{n}(q)$ par (3.19), sauf que la puissance de $d_{n}(q)$ est $A-1$ à la place de $A$. Comme pour $A=4$ et $r=1$, la série $\tilde{S}_{n}(q)$ est aussi identique avec le membre gauche de (4.7), cette « conjecture des dénominateurs » est vérifiée dans ce cas. Si elle l'est aussi pour $A=10$ et $r=2$, on pourra démontrer qu'au moins un des nombres $\zeta_{q}(3), \zeta_{q}(5), \zeta_{q}(7), \zeta_{q}(9)$ est irrationnel, et donc améliorer le résultat du Théorème 1.4. Bien que pour $A=4$ et $r=1$ la série $S_{n}^{[1]}(q)$ coïncide avec $\tilde{S}_{n}(q)$ (à un facteur négligeable près), une telle amélioration des dénominateurs n'a probablement pas lieu, en général, pour la série $S_{n}^{[\epsilon]}(q)$.

Remerciements. La recherche du premier auteur a eté partiellement supportée par la Fondation Autrichienne de la Recherche Scientifique FWF, contrat P12094-MAT, et par le Programme «Accroittre le potentiel humain de recherche» de la Commission Européenne, contrat HPRN-CT-2001-00272, 'Algebraic Combinatorics in Europe'.

La recherche du troisième auteur a eté supportée par une bourse «Alexander von Humboldt » et a eté partiellement supportée par la Fondation Russe pour la Recherche Fondamentale, contrat 03-01-00359. 


\section{Références}

1. R. ApÉRY, Irrationalité de $\zeta(2)$ et $\zeta(3)$, Astérisque 61 (1979), 11-13.

2. K. Ball et T. Rivoal, Irrationalité d'une infinité de valeurs de la fonction zêta aux entiers impairs, Invent. Math. 146(1) (2001), 193-207.

3. K. Barré-Siriex, G. Diaz, F. Gramain et G. Philibert, Une preuve de la conjecture de Mahler-Manin, Invent. Math. 124(1) (1996), 1-9.

4. E. V. Bedulev, On the linear independence of numbers over number fields, Mat. Zametki 64 (1998), 506-517 (en russe; trad. en anglais dans Math. Notes 64 (1998), 440-449).

5. D. Bertrand, Séries d'Eisenstein et transcendance, Bull. Soc. Math. France 104(3) (1976), 309-321.

6. F. Beukers, Padé-approximations in number theory, in Padé Approximation and its Applications, Amsterdam, 1980, pp. 90-99, Lecture Notes in Mathematics, vol. 888 (Springer, 1981).

7. P. Borwein, On the irrationality of certain series, Math. Proc. Camb. Phil. Soc. 112(1) (1992), 141-146.

8. P. Bundschun et K. VÄ̈̈nÄnen, Arithmetical investigations of a certain infinite product, Compositio Math. 91(2) (1994), 175-199.

9. S. Fischler, Irrationalité de valeurs de zêta (d'après Apéry, Rivoal, ...), in Séminaire Bourbaki 2002-2003, exposé no. 910, Astérisque 294 (2004), 27-62.

10. G. Gasper et M. Rahman, Basic hypergeometric series, Encyclopedia of Mathematics and its Applications, vol. 35 (Cambridge University Press, 1990).

11. L. A. Gutnik, Irrationality of some quantities that contain $\zeta(3)$, Acta Arith. 42(3) (1983), 255-264 (en russe; trad. en anglais dans Am. Math. Soc. Transl. (Ser. 2) 140 (1988), 45$55)$.

12. C. JADOT, Critères pour l'indépendance linéaire et algébrique, Thèse de Doctorat, Institut de Mathématiques de Jussieu (1996).

13. W. P. Johnson, The curious history of Faà di Bruno's formula, Am. Math. Mon. 109(3) (2002), 217-234.

14. M. Kaneko, N. Kurokawa et M. Wakayama, A variation of Euler's approach to values of the Riemann zeta function, Kyushu J. Math. 57(1) (2003), 175-192.

15. J. Lewis et D. Zagier, Period functions for Maass wave forms, I, Ann. Math. 153 (2002), 191-258.

16. Yu. V. Nesterenko, On the linear independence of numbers, Vestn. Moskov. Univ. Ser. I 1 (1985), 46-54 (en russe; trad. en anglais dans Mosc. Univ. Math. Bull. 40(1) (1985), 69-74).

17. Yu. V. Nesterenko, A few remarks on $\zeta(3)$, Mat. Zametki 59(6) (1996), 865-880 (en russe; trad. en anglais dans Math. Notes 59(6) (1996), 625-636).

18. Yu. V. Nesterenko, Modular functions and transcendence questions, Mat. Sb. 187(9) (1996), 65-96 (en russe; trad. en anglais dans Sb. Math. 187(9) (1996), 1319-1348).

19. P. Philippon, Une approche méthodique pour la transcendance et l'indépendance algébriques de valeurs de fonctions analytiques, J. Number Theory 64 (1997), 291-338.

20. K. Postelmans et W. Van Assche, Irrationality of $\zeta_{q}(1)$ and $\zeta_{q}(2)$, prépublication.

21. T. RivoAL, La fonction zêta de Riemann prend une infinité de valeurs irrationnelles aux entiers impairs, C. R. Acad. Sci. Paris Sér. I 331(4) (2000), 267-270.

22. T. RivonL, Propriétés diophantiennes des valeurs de la fonction zêta de Riemann aux entiers impairs, Thèse de Doctorat, Université de Caen (2001).

23. T. Rivoal, Irrationalité d'au moins un des neuf nombres $\zeta(5), \zeta(7), \ldots, \zeta(21)$, Acta Arith. 103(2) (2002), 157-167.

24. T. SCHNEIDER, Introduction aux nombres transcendants, traduit de l'allemand par P. Eymard (Gauthier-Villars, Paris, 1959).

25. J. P. Serre, Cours d'arithmétique (Presses Universitaires de France, Paris. 1970). 
26. R. P. Stanley, Enumerative combinatorics, vol. 1 (Wadsworth \& Brooks/Cole, Pacific Grove, CA, 1986) (réimprimé par Cambridge University Press, 1998).

27. T. TÖPfER, Über lineare Unabhängigkeit in algebraischen Zahlkörpern, Results Math. 25 (1994), 139-152.

28. W. VAN Assche, Little $q$-Legendre polynomials and irrationality of certain Lambert series, Ramanujan J. 5(3) (2001), 295-310.

29. W. Zudilin, Remarks on irrationality of q-harmonic series, Manuscr. Math. 107(4) (2002), 463-477.

30. W. ZudiLin, On the irrationality measure of a $q$-analogue of $\zeta(2)$, Mat. Sb. 193(8) (2002), 49-70 (en russe; trad. en anglais dans Sb. Math. 193(8) (2002), 1151-1172).

31. W. Zudilin, Diophantine problems for q-zeta values, Mat. Zametki 72(6) (2002), 936-940 (en russe; trad. en anglais dans Math. Notes 72(6) (2002), 858-862).

32. W. ZudiLin, On the functional transcendence of $q$-zeta values, Mat. Zametki 73(4) (2003), 629-630 (en russe; trad. en anglais dans Math. Notes 73(4) (2003), 588-589).

33. W. ZudiLin, Heine's basic transform and a permutation group for $q$-harmonic series, Acta Arith. 111(2) (2004), 153-164.

34. W. Zudilin, Arithmetic of linear forms involving odd zeta values, J. Théor. Nombres Bordeaux 16 (2004), 251-291. 
